\title{
PROJECTIVE RESOLUTIONS AND POINCARÉ DUALITY COMPLEXES
}

\author{
D. J. BENSON AND JON F. CARLSON
}

\begin{abstract}
Let $k$ be a field lof characteristic $p>0$ and let $G$ be a finite group. We investigate the structure of the cohomology ring $H^{*}(G, k)$ in relation to certain spectral sequences determined by systems of homogeneous parameters for the cohomology ring. Each system of homogeneous parameters is associated to a complex of projective $k G$-modules which is homotopically equivalent to a Poincare duality complex. The initial differentials in the hypercohomology spectral sequence of the complex are multiplications by the parameters, while the higher differentials are matric Massey products. If the cohomology ring is Cohen-Macaulay, then the duality of the complex assures that the Poincare series for the cohomology satisfies a certain functional equation. The structure of the complex also implies the existence of cohomology classes which are in relatively large degrees but are not in the ideal generated by the parameters. We consider several other questions concerned with the minimal projective resolutions and the convergence of the spectral sequence.
\end{abstract}

\section{INTRODUCTION}

For a finite dimensional algebra $A$, any finitely generated module is built from simple modules by a process of extensions. The process is regulated by a set of rules which are peculiar to the algebra. In general the rules can be very subtle and difficult to express in simple terms. Yet, they are recorded completely in any of several structures associated to the algebra and its module category. Three such structures are the form and constitution of the projective modules, the minimal projective resolutions of the simple modules, and the collection of extension groups Ext $_{A}^{n}$ between simple modules, together with the (Yoneda and Massey) product information. Of course, there may be difficulties translating from one structure to another. It may not be easy to construct projective resolutions even with full knowledge of the projective modules. Each of the structures has both advantages and disadvantages with respect to problems such as computability and interpretation of information. However, it is certainly true that each of these structures, if understood thoroughly, would provide a complete picture of the module theory for the algebra.

Each of the structures mentioned above has been studied extensively for many individual algebras and classes of algebras. We are particularly interested in group algebras of finite groups. In this case, the amount of information needed

Received by the editors May 1, 1990 and, in revised form, December 15, 1991.

1991 Mathematics Subject Classification. Primary 20J06, 20 C20.

The second author was partially supported by a grant from the NSF. 
to generate the structures is finite in the following sense. If $k$ is a field of characteristic $p$ and $G$ is a finite group then a theorem of Evens [13] says that the cohomology ring $H^{*}(G, k)$ is a finitely generated $k$-algebra. Also if $M$ is a finitely generated $k G$-module then $H^{*}(G, M)$ is finitely generated as a module over $H^{*}(G, k)$. From this it can be deduced that the minimal projective resolution of $M$ has a polynomial growth rate. But what do the minimal resolutions look like? How much information is really needed to generate them?

Although the subject of this paper is the cohomology rings of finite groups, the roots and motivation lie firmly within the area of representation theory, and in particular the attempt to understand minimal resolutions. The study grew out of the authors' work in [3] and [4], where the idea of using a homogeneous set of parameters to define filtrations on projective resolutions was first developed. In [4] we showed any module has a projective resolution which has the minimal polynomial growth rate, and which is formed by repeatedly splicing together certain finite complexes which are defined by the parameters. However, in general the resulting resolutions are not minimal, and we are left with the problem of understanding to what extent projectives may be stripped away to yield a smaller resolution. In $\S 9$ of [3], we answered this question for groups of $p$-rank two, provided certain conditions on the chain maps induced by the parameters are satisfied.

The primary purpose is to set up a framework for dealing with this problem in general. At present, we should honestly state that we open up more questions than we answer, but the theory shows some promise in that several extensions of the results have been obtained since the original manuscript for this paper was written. The study revolves around the investigation of finite complexes associated to systems of parameters in the cohomology ring. Each complex consists only of finitely generated projective modules, and is zero in all but a finite number of degrees. The complexes satisfy a Poincaré duality, and seem to encode an enormous amount of information about minimal resolutions, and in particular about the behaviour of the cohomology ring $H^{*}(G, k)$. We see, for example, that most finitely generated $k$-algebras cannot possibly be cohomology rings of finite groups. Although we work largely with coefficients in a field of characteristic $p$, the reader should notice that, with some modification, many of the techniques and results are applicable to the more general situation of cohomology with other coefficients.

After some generalities on chain complexes in $\S 2$, we introduce the finite complex associated to a system of parameters in $\S 3$. Using hypercohomology, we give a new proof in $\S 4$ of the basic result of [4], which states that the complexes consist of projective modules. Each complex looks homologically like a product of spheres with a free $G$-action. This analogy is taken further in $\S 5$, where we prove the Poincare duality with respect to the top homology class. We pass from information about the finite complex to information about minimal resolutions and cohomology by using the hypercohomology spectral sequence. This is explained in $\S 4$, where we also determine enough about the differentials in the sequence to begin to understand what is happening (Theorem 5.5).

There is one case in which we do obtain very striking and easily statable results. This is for groups whose cohomology rings are Cohen-Macaulay. Section 6 is devoted to this case, and among other things we prove the following. 
Theorem 1.1. Suppose that $H^{*}(G, k)$ is a Cohen-Macaulay ring. Let $r$ be the p-rank of $G$, which by a theorem of Quillen [21] is equal to the Krull dimension of $H^{*}(G, k)$. So we may choose a homogeneous set of parameters $\zeta_{1}, \ldots, \zeta_{r}$ (i.e., $H^{*}(G, k)$ is a finitely generated algebra over the polynomial subring $\left.k\left[\zeta_{1}, \ldots, \zeta_{r}\right]\right)$ with $\operatorname{deg}\left(\zeta_{i}\right) \geq 2$. Then the quotient

$$
H^{*}(G, k) /\left(\zeta_{1}, \ldots, \zeta_{r}\right)
$$

satisfies Poincaré duality in formal dimension $s=\sum_{i=1}^{r}\left(\operatorname{deg}\left(\zeta_{i}\right)-1\right)$. Moreover, the Poincaré series $P_{k}(t)=\sum_{i \geq 0} t^{i} \operatorname{dim}_{k} H^{i}(G, k)$, regarded as a rational function of $t$, satisfies the functional equation

$$
P_{k}(1 / t)=(-t)^{r_{p}(G)} P_{k}(t)
$$

One interpretation of this theorem is that the minimal projective resolution for the trivial module can be constructed by splicing together copies of the finite complex mentioned above.

Groups with abelian Sylow $p$-subgroups have cohomology rings which are Cohen-Macaulay. It also happens for some other groups. However it is important to realise that this is far from the generic case. Consider, for example, the following.

Proposition 1.2. If a finite group $G$ has maximal elementary abelian p-groups of different ranks, then $H^{*}(G, k)$ is not Cohen-Macaulay.

Hence, in the defining characteristic, the cohomology ring of a finite group of Lie type is usually not Cohen-Macaulay. The proof of the proposition follows easily from Quillen's Dimension Theorem [21], which asserts that the irreducible components of the maximal ideal spectrum of $H^{*}(G, k)$ are in oneone correspondence with the conjugacy classes of maximal elementary abelian $p$-subgroups of $G$, and that the dimension of each component is equal to the rank of the corresponding subgroup. The proof is completed by recalling that for a ring to be Cohen-Macaulay it is necessary for the components of the maximal ideal spectrum all to have the same dimension (see for example Matsumura [17, Theorem 17.3]). We should notice further that the converse of the proposition is false. Counterexamples include the semidihedral 2-groups $(p=2)$, and the extraspecial groups of order $p^{3}$ and exponent $p^{2}$ (for $p$ odd).

Another interpretation of Theorem 1.1 is that if $H^{*}(G, k)$ is a CohenMacaulay ring, then it is a Gorenstein ring (see Stanley [27, Theorem 5.5]). This is true not just in the usual (ungraded) sense, but in the sense that with the appropriate degree conventions, the canonical module is isomorphic to the ring as a graded module. Thus, for example, a polynomial ring is only Gorenstein in the graded sense if the generators are in degree one (cf. Corollary 6.6).

In the general case, where $H^{*}(G, k)$ is not necessarily Cohen-Macaulay, we can use the fact that the cohomology of an elementary abelian subgroup is Cohen-Macaulay to show that at least some part of the finite complex is visible in the cohomology of the group; namely the element of largest dimension. We call this element the last survivor. In $\S 7$, we prove the following theorem.

Theorem 1.3. If $\zeta_{1}, \ldots, \zeta_{r}$ is an irredundant homogeneous set of parameters for $H^{*}(G, k)$ with $\operatorname{deg}\left(\zeta_{i}\right)=n_{i} \geq 2$, then the quotient $H^{*}(G, k) /\left(\zeta_{1}, \ldots, \zeta_{r}\right)$ by 
the ideal generated by $\zeta_{1}, \ldots, \zeta_{r}$ has a nonzero element in degree $\sum_{i=1}^{r}\left(n_{i}-1\right)$. This element is a transfer from an elementary abelian subgroup.

To return to the representation theory, in $\S 8$ we show how to pass from a complex of projectives to a filtration on a projective module. In the case of the complexes introduced in $\S 4$, the $E_{2}$ term of the spectral sequence associated to this filtration is the Koszul complex. A distillation of the philosophy of the paper is expressed in this section.

Section 9 discusses some structural questions suggested by our constructions. We discuss to what extent projective modules may be eliminated from one version of the complexes described in $\S 4$. If the questions have a positive answer, then the Poincare series of the cohomology ring is given by a formula described at the end of the section. There is one situation in which these questions have a positive answer, which is formulated in the notion of a quasi-regular sequence in $\S 10$.

Finally, in $\S 11$ we show how certain secondary operations expressed in terms of matric Massey products are related to the differentials in the spectral sequence of $\S 4$.

\section{ChAIN COMPLEXES AND HYPERCOHOMOLOGY}

In this section we develop some generalities concerning chain complexes over finite groups. Most of the results in the section are known in some sense, and those that are not can be easily derived. However the notation and statements of the results are very important for the sections which follow.

Suppose $R$ is a commutative ring of coefficients and $\mathbf{C}$ and $\mathbf{D}$ are chain complexes of left $R$-modules, with differentials of degree -1 . We define a new chain complex $\operatorname{Hom}_{R}(\mathbf{C}, \mathbf{D})$ with

$$
\operatorname{Hom}_{R}(\mathbf{C}, \mathbf{D})_{n}=\bigoplus_{i+n=j} \operatorname{Hom}_{R}\left(C_{i}, D_{j}\right)
$$

with differential

$$
\partial_{n}: \operatorname{Hom}_{R}(\mathbf{C}, \mathbf{D})_{n} \rightarrow \operatorname{Hom}_{R}(\mathbf{C}, \mathbf{D})_{n-1}
$$

defined so that

$$
\partial_{j}(f(x))=\left(\partial_{n} f\right)(x)+(-1)^{n} f\left(\partial_{i}(x)\right)
$$

for $f \in \operatorname{Hom}_{R}\left(C_{i}, D_{j}\right)$. In other words, $\partial_{n}$ is defined by

$$
\left(\partial_{n} f\right)(x)=\partial_{j}(f(x))-(-1)^{n} f\left(\partial_{i}(x)\right) .
$$

This may be expressed by writing $\partial f=[\partial, f]$. With this definition, an element $f \in \operatorname{Hom}_{R}(\mathbf{C}, \mathbf{D})_{0}$ is a map of chain complexes if and only if $f$ is a cycle $(\partial f=0)$. Moreover, $f$ and $g$ are homotopic if and only if $f-g$ is a boundary.

If $M$ is a left $R$-module, we write $M[n]$ for the chain complex consisting of $M$ in degree $n$ and zero elsewhere. We also write $M$ for the complex $M[0]$ consisting of $M$ in degree 0 and zero elsewhere. If $\mathbf{C}$ is a chain complex of left $R$-modules, we write $\mathbf{C}[n]$ for $R[n] \otimes_{R} \mathbf{C}$. Namely we have $(\mathbf{C}[n])_{i+n}=(\mathbf{C})_{i}$. Note that the differential in $\mathbf{C}[n]$ is $(-1)^{n}$ times the differential in $\mathbf{C}$. 
The dual of $\mathbf{C}$ is the chain complex $\operatorname{Hom}_{R}(\mathbf{C}, R)$. Note that the differential on the dual is given by $\left(\partial_{n} f\right)(x)=(-1)^{n-1} f\left(\partial_{-n+1}(x)\right)$. With these sign conventions, evaluation is a map of chain complexes

$$
\operatorname{Hom}_{R}(\mathbf{C}, R) \otimes_{R} \mathbf{C} \rightarrow R, \quad f \otimes x \mapsto f(x) .
$$

Finally, we regard chain complexes and cochain complexes as being the same thing. Namely, if $\left(C_{n}, \partial_{n}\right)$ is a chain complex, then setting $C^{n}=C_{-n}$ and $\delta^{n}=\partial_{-n}$, we have a cochain complex $\left(C^{n}, \delta^{n}\right)$. In the end, whether we regard a particular complex as a chain complex or a cochain complex often depends on where it came from.

We now discuss Ext for chain complexes. This is sometimes also called hypercohomology; see for example Cartan and Eilenberg [9].

Definition 2.1. Suppose that $\mathrm{C}$ is a chain complex of left $R G$-modules, bounded below. Then a projective resolution of $\mathbf{C}$ is a chain complex $\mathbf{P}$ of projective left $R G$-modules, bounded below, together with a map of chain complexes $\mathbf{P} \rightarrow \mathrm{C}$ which is an isomorphism on homology.

Note that in the case $\mathbf{C}=M$ is a module concentrated in degree zero, this agrees with the usual definition of a projective resolution of a module. Existence of projective resolutions is easy to prove inductively using the definition of projective modules. Alternatively, one may tensor $\mathbf{C}$ with a projective resolution of the trivial $R G$-module $R$. The usual comparison theorem holds for projective resolutions of chain complexes. If $R$ is a field (or more generally a local ring) then minimal resolutions exist and are unique, in the following sense. The resolution, as a complex of $R G$-modules, is uniquely determined, but the augmentation map $\mathbf{P} \rightarrow \mathbf{C}$ is only unique up to homotopy. Minimal resolutions are characterised by the property that the image of each term is contained in the radical of the next.

If $\mathbf{D}$ is another chain complex of left $R G$-modules, bounded above, we may define

$$
\operatorname{Ext}_{R G}^{n}(\mathbf{C}, \mathbf{D})=H^{n}\left(\operatorname{Hom}_{R G}(\mathbf{P}, \mathbf{D})\right) .
$$

(Note that we are regarding this chain complex as a cochain complex by negating the degrees.) If $\mathbf{I}$ is an injective resolution of $\mathbf{D}$ (defined dually to the above definition) then

$$
\operatorname{Hom}_{R}(\mathbf{P}, \mathbf{D}) \rightarrow \operatorname{Hom}_{R}(\mathbf{P}, \mathbf{I}) \leftarrow \operatorname{Hom}_{R}(\mathbf{C}, \mathbf{I})
$$

are homotopy equivalences, and so

$$
\operatorname{Ext}_{R G}^{n}(\mathbf{C}, \mathbf{D}) \cong H^{n}\left(\operatorname{Hom}_{R G}(\mathbf{C}, \mathbf{I})\right) .
$$

We write $H^{n}(G, \mathbf{C})$ for $\operatorname{Ext}_{R G}^{n}(R, \mathbf{C})$. Note that

$$
\operatorname{Ext}_{R G}^{n}(\mathbf{C}, \mathbf{D}) \cong H^{n}\left(G, \operatorname{Hom}_{R}(\mathbf{C}, \mathbf{D})\right) .
$$


If $\mathbf{D}$ is bounded (i.e., bounded both above and below), then maps from $\mathbf{P}$ to $\mathbf{D}$ may be lifted to maps to a projective resolution of $\mathbf{D}$. So if $\mathbf{E}$ is bounded above, then composition of maps in Hom gives rise to Yoneda composition

$$
\mathrm{Ext}_{R G}^{*}(\mathbf{D}, \mathbf{E}) \otimes \mathrm{Ext}_{R G}^{*}(\mathbf{C}, \mathbf{D}) \rightarrow \mathrm{Ext}_{R G}^{*}(\mathbf{C}, \mathbf{E}),
$$

which agrees with the usual Yoneda composition in case $\mathbf{C}, \mathbf{D}$ and $\mathbf{E}$ are modules concentrated in degree zero. Indeed, this was part of the original motivation for the definition of the derived category, which is really what we are using in disguise (see [11, Appendix 1]).

The tensor product map

$$
\operatorname{Hom}_{R G}(\mathbf{C}, \mathbf{D}) \otimes \operatorname{Hom}_{R G}\left(\mathbf{C}^{\prime}, \mathbf{D}^{\prime}\right) \rightarrow \operatorname{Hom}_{R G}\left(\mathbf{C} \otimes \mathbf{C}^{\prime}, \mathbf{D} \otimes \mathbf{D}^{\prime}\right)
$$

gives rise to a cup product map

$$
\begin{aligned}
\operatorname{Ext}_{R G}^{*}(\mathbf{C}, \mathbf{D}) \otimes \operatorname{Ext}_{R G}^{*}\left(\mathbf{C}^{\prime}, \mathbf{D}^{\prime}\right) & \rightarrow \operatorname{Ext}_{R G}^{*}\left(\mathbf{C} \otimes \mathbf{C}^{\prime}, \mathbf{D} \otimes \mathbf{D}^{\prime}\right), \\
\zeta \otimes \eta & \mapsto \zeta \cup \eta .
\end{aligned}
$$

For convenience of notation, we sometimes also denote the cup product by juxtaposition.

Lemma 2.2. If $\zeta \in \operatorname{Ext}_{R G}^{m}(\mathbf{C}, \mathbf{D}), \eta \in \operatorname{Ext}_{R G}^{n}\left(\mathbf{C}^{\prime}, \mathbf{D}^{\prime}\right)$ then the cup product

$$
\zeta \cup \eta \in \operatorname{Ext}_{R G}^{m+n}\left(\mathbf{C} \otimes \mathbf{C}^{\prime}, \mathbf{D} \otimes \mathbf{D}^{\prime}\right)
$$

is equal to the Yoneda composite of

$$
\zeta \otimes \mathrm{id}_{\mathbf{D}^{\prime}} \in \mathrm{Ext}_{R G}^{m}\left(\mathbf{C} \otimes \mathbf{D}^{\prime}, \mathbf{D} \otimes \mathbf{D}^{\prime}\right)
$$

and

$$
\operatorname{id}_{\mathbf{C}} \otimes \eta \in \operatorname{Ext}_{R G}^{n}\left(\mathbf{C} \otimes \mathbf{C}^{\prime}, \mathbf{C} \otimes \mathbf{D}^{\prime}\right) .
$$

Proof. This follows easily from the corresponding (obvious) statement at the level of Hom .

Since tensor products are graded commutative, so are cup products, in the sense that the following diagram commutes.

$$
\begin{array}{rlc}
\operatorname{Ext}_{R G}^{m}(\mathbf{C}, \mathbf{D}) \otimes \operatorname{Ext}_{R G}^{n}\left(\mathbf{C}^{\prime}, \mathbf{D}^{\prime}\right) & \rightarrow & \operatorname{Ext}_{R G}^{m+n}\left(\mathbf{C} \otimes \mathbf{C}^{\prime}, \mathbf{D} \otimes \mathbf{D}^{\prime}\right) \\
\downarrow \cong & & \downarrow \cong \\
\operatorname{Ext}_{R G}^{n}\left(\mathbf{C}^{\prime}, \mathbf{D}^{\prime}\right) \otimes \operatorname{Ext}_{R G}^{m}(\mathbf{C}, \mathbf{D}) & \rightarrow & \operatorname{Ext}_{R G}^{m+n}\left(\mathbf{C}^{\prime} \otimes \mathbf{C}, \mathbf{D}^{\prime} \otimes \mathbf{D}\right)
\end{array}
$$

But in general Yoneda products are not graded commutative. So $\operatorname{Ext}_{R G}^{*}(\mathbf{C}, \mathbf{C})$ is in general a noncommutative graded ring and a module over the graded commutative ring $H^{*}(G, R)=\operatorname{Ext}_{R G}^{*}(R, R)$.

Regarding $\operatorname{Hom}_{R G}(\mathbf{C}, \mathbf{I})$ as a double complex, we obtain a spectral sequence, called the hypercohomology spectral sequence

$$
E_{2}^{p q}=\operatorname{Ext}_{R G}^{p}\left(H_{q}(\mathbf{C}), \mathbf{D}\right) \Rightarrow \operatorname{Ext}_{R G}^{p+q}(\mathbf{C}, \mathbf{D}) .
$$

Similarly, regarding $\operatorname{Hom}_{R G}(\mathbf{P}, \mathbf{D})$ as a double complex, we have a spectral sequence

$$
E_{2}^{p q}=\operatorname{Ext}_{R G}^{p}\left(\mathbf{C}, H_{-q}(\mathbf{D})\right) \Rightarrow \operatorname{Ext}_{R G}^{p+q}(\mathbf{C}, \mathbf{D}) .
$$


Theorem 2.4. Suppose that $\mathbf{C}$ and $\mathbf{D}$ are chain complexes of left $R G$-modules. If $\operatorname{Hom}_{R}(\mathbf{C}, \mathbf{D})$ is a Noetherian $R$-module (and hence nonzero in only finitely many degrees) then $\operatorname{Ext}_{R G}^{*}(\mathbf{C}, \mathbf{D})$ is Noetherian as a module over $H^{*}(G, R)$.

Proof. The proof given in Evens [13] for modules generalises directly to chain complexes.

Corollary 2.5. If $R$ is a commutative Noetherian ring, and $\mathbf{C}$ and $\mathbf{D}$ are complexes of left $R G$-modules, each finitely generated as an $R$-module, then the ring $\operatorname{Ext}_{R G}^{*}(\mathbf{C}, \mathbf{C})$ is a finitely generated $R$-algebra, and $\operatorname{Ext}_{R G}^{*}(\mathbf{C}, \mathbf{D})$ is a finitely generated $\mathrm{Ext}_{R G}^{*}(\mathbf{C}, \mathbf{C})$-module.

Proof. It is a finitely generated module over the finitely generated $R$-algebra $H^{*}(G, R)$, and the products are compatible by Lemma 2.2 .

Proposition 2.6. Suppose that $k$ is a field and $\mathbf{C}$ has finite total dimension over $k$. If $\operatorname{Ext}_{k G}^{n}(\mathbf{C}, \mathbf{C})=0$ for all $n$ sufficiently large, then $\mathbf{C}$ has a finite projective resolution.

Proof. If $\mathbf{D}$ is another complex of finite total dimension over $k$, then by Lemma 2.2 the action of $H^{*}(G, k)$ on $\operatorname{Ext}_{k G}^{*}(\mathbf{C}, \mathbf{D})$ factors as the map

$$
H^{*}(G, k)=\operatorname{Ext}_{k G}^{*}(k, k) \rightarrow \operatorname{Ext}_{k G}^{*}(\mathbf{C}, \mathbf{C})
$$

followed by Yoneda composition. Since $\operatorname{Ext}_{k G}^{*}(\mathbf{C}, \mathbf{D})$ is finitely generated as a module over $H^{*}(G, k)$ by Theorem 2.4 , it is finitely generated as a module over $\operatorname{Ext}_{k G}^{*}(\mathbf{C}, \mathbf{C})$. The latter has finite total dimension by the hypothesis, and so $\operatorname{Ext}_{k G}^{n}(\mathbf{C}, \mathbf{D})=0$ for all $n$ sufficiently large.

Now let $\mathbf{P}$ be the minimal resolution of $\mathbf{C}$. Then for each simple $k G$-module $S$,

$$
\operatorname{Ext}_{k G}^{n}(\mathbf{C}, S)=\operatorname{Hom}_{k G}\left(P_{n}, S\right)
$$

is nonzero for only finitely many $n$. It follows that $P_{n}=0$ for all but finitely many $n$.

\section{FRom PARAMETERS TO COMPLEXES}

In [4], we introduced a construction for projective resolutions using as the basic building block a certain finite complex of projective modules. These complexes and others, which can be similarly derived, are the main focus of the paper. We present here a summary of the construction and introduce some ideas and notations which are needed later. The proof we gave that the modules in the complexes are projective used the machinery of varieties for modules, and in particular depended on theorems of Quillen [21]. In $\S 4$ we give an alternative proof avoiding this machinery.

The construction goes as follows. Suppose $\zeta \in H^{n}(G, R) \cong \operatorname{Ext}_{R G}^{n}(R, R)$ with $n \geq 2$. We choose a cocycle $\hat{\zeta}: \tilde{\Omega}^{n} R \rightarrow R$ representing $\zeta$, where $\tilde{\Omega}^{n} R$ is the $n$th kernel in a projective resolution $\mathbf{P}$ of $R$ as an $R G$-module. By making P large enough, we may assume $\hat{\zeta}$ is surjective. We denote its kernel by $L_{\zeta}$, 
and form the pushout diagram

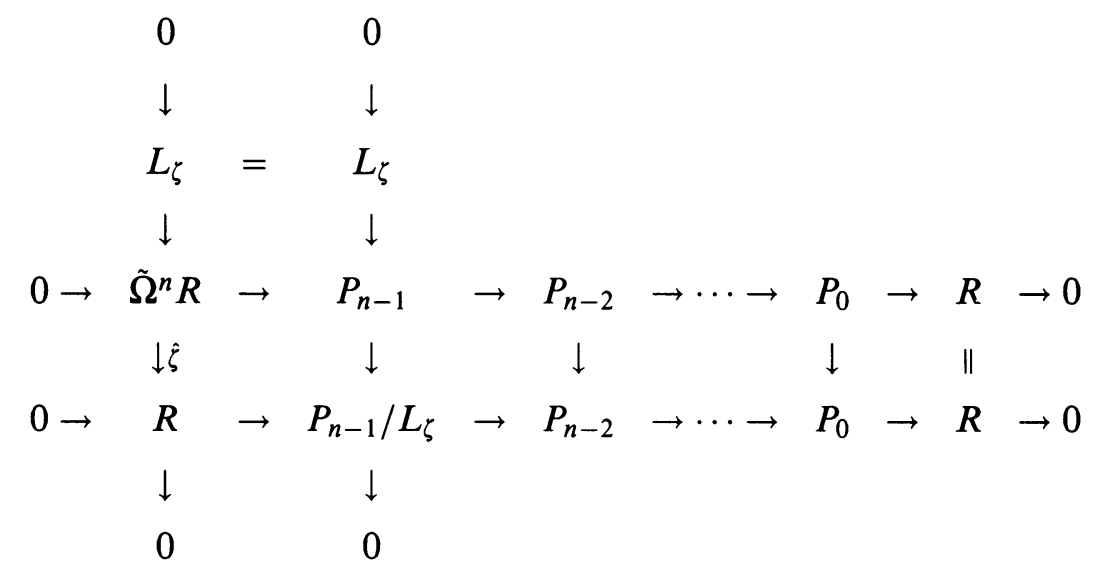

The bottom row of this diagram is an $n$-fold extension representing the element $\zeta \in \operatorname{Ext}_{R G}^{n}(R, R)$. We denote by $\mathbf{C}_{\zeta}$ the chain complex

$$
0 \rightarrow P_{n-1} / L_{\zeta} \rightarrow P_{n-2} \rightarrow \cdots \rightarrow P_{0} \rightarrow 0
$$

formed by truncating the bottom row of this diagram. Thus we have

$$
H_{i}\left(\mathbf{C}_{\zeta}\right) \cong \begin{cases}R & \text { if } i=0, n-1 \\ 0 & \text { otherwise. }\end{cases}
$$

We write $\tilde{\zeta}$ for the generator of degree $n-1$, and 1 for the generator of degree zero.

The reason why we demand that $\operatorname{deg}(\zeta) \geq 2$ is that if $\operatorname{deg}(\zeta)=1$ then $\mathbf{C}_{\zeta}$ is a complex consisting of a single module in degree zero, which is the extension of $R$ by $R$ corresponding to $\zeta$.

The complex $\mathbf{C}_{\zeta}$ should be thought of as a sort of algebraic analogue of a sphere with $G$-action, with $\zeta$ being the transgression of the fundamental class of the sphere.

We also write $\mathbf{C}_{\zeta}^{(\infty)}$ for the chain complex

$$
\cdots \rightarrow P_{1} \rightarrow P_{0} \rightarrow P_{n-1} / L_{\zeta} \rightarrow P_{n-2} \rightarrow \cdots \rightarrow P_{1} \rightarrow P_{0} \rightarrow 0
$$

obtained by splicing together infinitely many copies of $\mathbf{C}_{\zeta}$ in positive degree. It is an exact complex except in degree zero, where the homology is $R$. This is the complex used in [4].

Lemma 3.1. Suppose that $\mathbf{D}$ is a bounded chain complex of RG-modules. In the two-row spectral sequence

$$
E_{2}^{p q}(\zeta)=\operatorname{Ext}_{R G}^{p}\left(H_{q}\left(\mathbf{C}_{\zeta}\right), \mathbf{D}\right) \Rightarrow \operatorname{Ext}_{R G}^{p+q}\left(\mathbf{C}_{\zeta}, \mathbf{D}\right)
$$

the differential $d_{n}$ is given by

$$
d_{n}(\alpha . \tilde{\zeta})=\alpha . \zeta \in E_{n}^{p+n, 0}(\zeta)=H^{p+n}(G, \mathbf{D}) .
$$


Proof. We first remark that since $H_{q}\left(\mathbf{C}_{\zeta}\right)=R$ if $q=0$ or $n-1$ and is zero elsewhere, we have

$$
E_{2}^{p q}(\zeta)= \begin{cases}H^{p}(G, \mathbf{D}) & \text { if } q=0 \text { or } n-1 \\ 0 & \text { otherwise. }\end{cases}
$$

So the only possible nonzero differential is $d_{n}$.

The differential $d_{n}$ may be obtained as follows. We have a short exact sequence of chain complexes

$$
0 \rightarrow \mathbf{C}_{\zeta} \rightarrow \mathbf{C}_{\zeta}^{(\infty)} \rightarrow \mathbf{C}_{\zeta}^{(\infty)}[n] \rightarrow 0
$$

where $[n]$ indicates degree shift. If $\mathbf{P}$ is a projective resolution of $R$ as an $R G$-module then we have a short exact sequence

$$
0 \rightarrow \mathbf{P} \otimes \mathbf{C}_{\zeta} \rightarrow \mathbf{P} \otimes \mathbf{C}_{\zeta}^{(\infty)} \rightarrow \mathbf{P} \otimes \mathbf{C}_{\zeta}^{(\infty)}[n] \rightarrow 0
$$

and hence a short exact sequence of cochain complexes

$$
\begin{aligned}
0 & \rightarrow \operatorname{Hom}_{R G}\left(\mathbf{P} \otimes \mathbf{C}_{\zeta}^{(\infty)}, \mathbf{D}\right)[-n] \rightarrow \operatorname{Hom}_{R G}\left(\mathbf{P} \otimes \mathbf{C}_{\zeta}^{(\infty)}, \mathbf{D}\right) \\
& \rightarrow \operatorname{Hom}_{R G}\left(\mathbf{P} \otimes \mathbf{C}_{\zeta}, \mathbf{D}\right) \rightarrow 0 .
\end{aligned}
$$

Now $\mathbf{P} \otimes \mathbf{C}_{\zeta}^{(\infty)}$ is again a projective resolution of $R$ as an $R G$-module, so the long exact sequence in cohomology of this short exact sequence of cochain complexes is

$$
\cdots \rightarrow \operatorname{Ext}_{R G}^{r-1}\left(\mathbf{C}_{\zeta}, \mathbf{D}\right) \rightarrow H^{r-n}(G, \mathbf{D}) \stackrel{d_{n}}{\rightarrow} H^{r}(G, \mathbf{D}) \rightarrow \operatorname{Ext}_{R G}^{r}\left(\mathbf{C}_{\zeta}, \mathbf{D}\right) \rightarrow \cdots
$$

This is the long exact sequence associated to the above two-row spectral sequence, and so the marked homomorphism is $d_{n}$.

To identify this map as multiplication by $\zeta$, we argue as follows. In general, multiplication by $\zeta$ may be thought of in the following way. A cocycle representing the element $\zeta$ lifts to a map of projective resolutions $\mathbf{P} \rightarrow \mathbf{P}[n]$. If $\alpha \in H^{r-n}(G, \mathbf{D})$ is represented by a chain map $\mathbf{P} \rightarrow \mathbf{D}[r-n]$ then the product $\alpha . \zeta$ is represented by shifting and composing

$$
\mathbf{P} \rightarrow \mathbf{P}[n] \rightarrow \mathbf{D}[r] .
$$

So all we need check is that the map of resolutions

$$
\mathbf{P} \otimes \mathbf{C}_{\zeta}^{(\infty)} \rightarrow \mathbf{P} \otimes \mathbf{C}_{\zeta}^{(\infty)}[n]
$$

given above represents the element $\zeta$ in cohomology. But this follows from the diagram

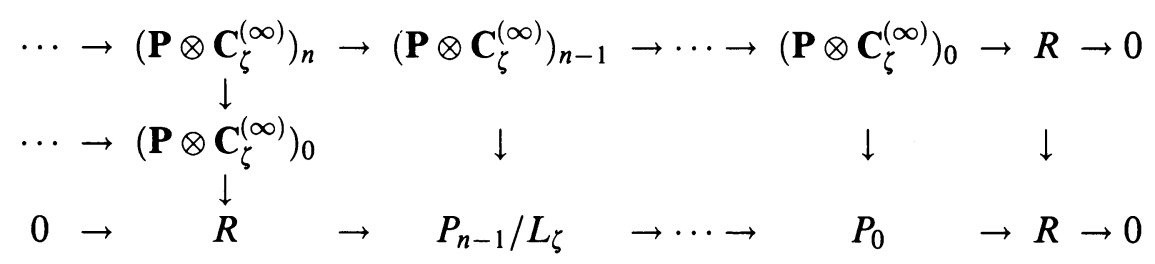

since the bottom row is an exact sequence representing $\zeta$. $\square$ 
Remark 3.2. There is a way of rewriting the above two-row spectral sequence in such a way that the two rows are adjacent and the differentials are unchanged. Namely, we replace the complex $\mathbf{C}_{\zeta}$ by the truncated complex

$$
\mathbf{B}_{\zeta}: 0 \rightarrow P_{n-1} / L_{\zeta} \rightarrow P_{n-2} \rightarrow 0
$$

whose homology is $k$ in degree one and $\Omega^{n-2} k$ in degree zero.

If $\zeta_{1}, \ldots, \zeta_{r} \in H^{*}(G, R)$ are homogeneous elements of degree $\geq 2$, then we form the complex $\mathbf{C}=\mathbf{C}_{\zeta_{1}} \otimes \cdots \otimes \mathbf{C}_{\zeta_{r}}$. The Künneth formula shows that $H_{*}(\mathbf{C})$ has a basis consisting of elements of the form $x_{1} \otimes \cdots \otimes x_{r}$ where each $x_{i}$ is equal to either 1 or $\tilde{\zeta}_{i}$. We also write $\tilde{\zeta}_{i}$ for the element $1 \otimes \cdots \otimes \tilde{\zeta}_{i} \otimes \cdots \otimes 1$ of $H_{*}(\mathbf{C})$. The complex $\mathbf{C}$ and the related complex $\mathbf{B}=\mathbf{B}_{\zeta_{1}} \otimes \cdots \otimes \mathbf{B}_{\zeta_{r}}$ (see $\S 8)$ are among the principal objects of study of this paper.

We are particularly interested in this construction in the following situation. If $R$ is Noetherian and $\mathbf{D}$ is a bounded chain complex of finitely generated $R G$-modules, then by Theorem $2.4, H^{*}(G, \mathrm{D})$ is a Noetherian module for $H^{*}(G, R)$. We say that elements $\zeta_{1}, \ldots, \zeta_{r} \in H^{*}(G, R)$ form a homogeneous set of parameters (h.s.o.p.) for $H^{*}(G, \mathbf{D})$ if $H^{*}(G, \mathbf{D})$ is Noetherian as a module for the subring of $H^{*}(G, R)$ generated by $\zeta_{1}, \ldots, \zeta_{r}$. In case $\mathbf{D}=R$, we simply speak of an h.s.o.p. for $H^{*}(G, R)$.

In case $R=k$ is a field, the number $r$ of parameters is at least the Krull dimension of the $H^{*}(G, k)$-module $H^{*}(G, \mathbf{D})$. Equality holds if and only if $\zeta_{1}, \ldots, \zeta_{r}$ generate a polynomial subring $k\left[\zeta_{1}, \ldots, \zeta_{r}\right]$ of the quotient of $H^{*}(G, k)$ by the annihilator of $H^{*}(G, \mathbf{D})$. In this case, we say that $\zeta_{1}, \ldots, \zeta_{r}$ form an irredundent h.s.o.p. The Noether normalisation lemma for Noetherian graded modules over a finitely generated commutative $k$-algebra says that an irredundent h.s.o.p. always exists. Geometrically, it is the same as saying that the homogeneous hypersurfaces $V_{G}\left(\zeta_{i}\right)$ defined by the $\zeta_{i}$ in the maximal ideal spectrum $V_{G}=\max H^{\text {even }}(G, k)$ intersect in the origin, and the number of hypersurfaces is exactly equal to the dimension of $V_{G}$.

The reader should beware at this stage that most texts on commutative algebra assume strict commutativity $x y=y x$. In our situation we have the sign conventions $x y=(-1)^{\operatorname{deg}(x) \operatorname{deg}(y)} y x$. One can check that the usual theorems of commutative algebra are also true in this situation after trivial alterations to the proofs.

\section{THE HYPERCOHOMOLOGY SPECTRAL SEQUENCE}

Let $\zeta_{1}, \ldots, \zeta_{r}$ be an h.s.o.p. for $H^{*}(G, k)$ with $\operatorname{deg}\left(\zeta_{i}\right)=n_{i} \geq 2$. We assume for convenience that the $n_{i}$ are even if $p$ is odd, since in this case elements of odd degree square to zero. We set $\mathbf{C}=\mathbf{C}_{\zeta_{1}} \otimes \cdots \otimes \mathbf{C}_{\zeta_{r}}$ as in the last section.

Suppose that $\mathbf{D}$ is a bounded chain complex of finitely generated $k G$ modules. Then from (2.3) we have a hypercohomology spectral sequence

$$
E_{2}^{p q}=\operatorname{Ext}_{k G}^{p}\left(H_{q}(\mathbf{C}), \mathbf{D}\right) \Rightarrow \operatorname{Ext}_{k G}^{p+q}(\mathbf{C}, \mathbf{D}) .
$$

In this spectral sequence, the $E_{2}$ page is a tensor product

$$
E_{2}^{p q} \cong H^{p}(G, \mathbf{D}) \otimes H^{q}\left(\operatorname{Hom}_{k}(\mathbf{C}, k)\right)
$$


and $H^{*}\left(\operatorname{Hom}_{k}(\mathbf{C}, k)\right)$ is an exterior algebra $\Lambda^{*}\left(\tilde{\zeta}_{1}, \ldots, \tilde{\zeta}_{r}\right)$ on classes $\tilde{\zeta}_{i}$ of degree $n_{i}-1$.

It should be remarked that in many cases the spectral sequence admits a product structure. Suppose that the complex $\mathbf{C}$ admits a diagonal approximation $\mathbf{C} \rightarrow \mathbf{C} \otimes \mathbf{C}$. There is always a diagonal approximation on a projective resolution of $k$. Hence the spectral sequence with $\mathbf{D}=k$ (concentrated in degree zero)

$$
H^{p}(G, k) \otimes H^{q}\left(\operatorname{Hom}_{k}(\mathbf{C}, k)\right) \Rightarrow H^{p+q}\left(\operatorname{Hom}_{k G}(\mathbf{C}, k)\right)
$$

has a ring structure over which the spectral sequence

$$
H^{p}(G, \mathbf{D}) \otimes H^{q}\left(\operatorname{Hom}_{k}(\mathbf{C}, k)\right) \Rightarrow H^{p+q}\left(\operatorname{Hom}_{k G}(\mathbf{C}, \mathbf{D})\right)
$$

is a module.

In $\S 5$ it is shown that $\mathbf{C}$ satisfies Poincare duality of formal dimension $s=$ $\sum_{i=1}^{r}\left(n_{i}-1\right)$, so that instead of using $\mathbf{C}_{\zeta_{i}}$ we may use $\operatorname{Hom}_{k}\left(\mathbf{C}_{\zeta_{i}}, k\right)\left[n_{i}-1\right]$. This turns out to be more convenient from the point of view of the diagonal approximation. This is because while $\mathbf{C}_{\zeta_{i}}$ usually does not admit a diagonal approximation, it was shown in [7] that $\operatorname{Hom}_{k}\left(\mathbf{C}_{\zeta_{i}}, k\right)\left[n_{i}-1\right]$ admits a diagonal approximation for which the augmentation is a counit if and only if $\zeta_{i}$ annihilates $\operatorname{Ext}_{k G}^{*}\left(L_{\zeta_{i}}, L_{\zeta_{i}}\right)$. By tensoring and using Poincaré duality, we obtain a diagonal approximation $\mathbf{C} \rightarrow \mathbf{C} \otimes \mathbf{C}$. It is also shown in [7] that as long as $p$ is odd, $\zeta_{i}$ always annihilates $\operatorname{Ext}_{k G}^{*}\left(L_{\zeta_{i}}, L_{\zeta_{i}}\right)$, so that the above spectral sequences have a multiplicative structure. For $p=2$, the situation is less clear.

Now we can use the maps of spectral sequences

$$
\begin{array}{rccc}
E_{2}^{p q}\left(\zeta_{i}\right)= & \operatorname{Ext}_{k G}^{p}\left(H_{q}\left(\mathbf{C}_{\zeta_{i}}\right), k\right) & \Rightarrow & \operatorname{Ext}_{k G}^{p+q}\left(\mathbf{C}_{\zeta_{i}}, k\right) \\
\downarrow & & & \downarrow \\
E_{2}^{p q}= & \operatorname{Ext}_{k G}^{p}\left(H_{q}(\mathbf{C}), k\right) & \Rightarrow & \operatorname{Ext}_{k G}^{p+q}(\mathbf{C}, k)
\end{array}
$$

to see, using Lemma 3.1 , that $d_{n_{i}}\left(\tilde{\zeta}_{i}\right)=\zeta_{i}$ in $E_{n_{i}}^{* *}$. In the case where the spectral sequence has a multiplicative structure, this determines the differentials on all elements of $E_{*}^{0 *}$, and shows that the spectral sequence is a sort of staggered version of the Koszul complex, together with possibly some further differentials. See Serre [25, Chapter IV], Matsumura [17, §16], or Carlson [8, §2] for discussions of the Koszul complex. We may make this into an actual Koszul complex without any staggering by using Remark 3.2. Namely, instead of $\mathbf{C}$ we use the complex $\mathbf{B}=\mathbf{B}_{\zeta_{1}} \otimes \cdots \otimes \mathbf{B}_{\zeta_{r}}$. In this case, we have $\tilde{\zeta}_{i} \in E_{2}^{*, 1}$ and $d_{2}\left(\tilde{\zeta}_{i}\right)=\zeta_{i} \in E_{2}^{*, 0}$, so that in this case

$$
E_{3}^{*, j}=\operatorname{Tor}_{j}^{R\left[\zeta_{1}, \ldots, \zeta_{r}\right]}\left(R, H^{*}(G, R)\right)
$$

In particular, if $H^{*}(G, R) /\left(\zeta_{1}, \ldots, \zeta_{r}\right)$ is a finitely generated $R$-module then $E_{3}^{* *}$ is also a finitely generated $R$-module and hence so also is $E_{\infty}^{* *}$. See $\S 8$ for more details.

Even when a spectral sequence such as given above does not have a multiplicative structure, it still has the structure of an $H^{*}(G, k)$-module, and much of the same information can be obtained about the differentials by considering maps of spectral sequences as follows. Suppose as above that $\mathbf{D}$ is a bounded 
chain complex of $R G$-modules. Then we have maps of spectral sequences

$$
\begin{array}{cccc}
E_{2}^{p q}= & \operatorname{Ext}_{k G}^{p}\left(H_{q}(\mathbf{C}), \mathbf{D}\right) & \Rightarrow & \operatorname{Ext}_{k G}^{p+q}(\mathbf{C}, \mathbf{D}) \\
\uparrow & \uparrow \\
E_{2}^{p q}\left(\zeta_{i_{1}}, \ldots, \zeta_{i_{t}}\right)=\operatorname{Ext}_{k G}^{p}\left(H_{q}\left(\mathbf{C}_{\zeta_{i_{1}}} \otimes \cdots \otimes \mathbf{C}_{\zeta_{i_{t}}}\right), \mathbf{D}\right) \Rightarrow \operatorname{Ext}_{k G}^{p+q}\left(\mathbf{C}_{\zeta_{i_{1}}} \otimes \cdots \otimes \mathbf{C}_{\zeta_{i_{t}}}, \mathbf{D}\right) \\
\downarrow & & \downarrow \\
E_{2}^{p, q-\ell}\left(\zeta_{i_{t}}\right)= & \operatorname{Ext}_{k G}^{p}\left(H_{q-\ell}\left(\mathbf{C}_{\zeta_{i_{t}}}\right), \mathbf{D}\right) & \Rightarrow & \operatorname{Ext}_{k G}^{p+q}\left(\mathbf{C}_{\zeta_{i_{t}}}, \mathbf{D}\right)
\end{array}
$$

(where $\ell=\sum_{j=1}^{t-1}\left(n_{i_{j}}-1\right)$ ). They are derived from the maps of complexes

$$
\mathbf{C} \rightarrow \mathbf{C}_{\zeta_{i_{1}}} \otimes \cdots \otimes \mathbf{C}_{\zeta_{i_{t}}} \leftarrow \mathbf{C}_{\zeta_{i_{t}}}[\ell]
$$

We deduce from this that if $\alpha \cdot \tilde{\zeta}_{i_{1}} \ldots \tilde{\zeta}_{i_{t}}$ survives to $E_{n_{t}}$ then the coefficient of $\tilde{\zeta}_{i_{1}} \ldots \tilde{\zeta}_{i_{t-1}}$ in $d_{n_{t}}\left(\alpha . \tilde{\zeta}_{i_{1}} \ldots \tilde{\zeta}_{i_{t}}\right)$ is $\alpha \zeta_{i_{t}}$, with the appropriate sign, as required.

Theorem 4.1. Suppose that $R$ is a field, or the ring of integers in an algebraic number field, or one of its localisations or completions. If $\zeta_{1}, \ldots, \zeta_{r}$ is an h.s.o.p. for $H^{*}(G, R)$ with $\operatorname{deg}\left(\zeta_{i}\right)=n_{i} \geq 2$, then the tensor product

$$
\mathbf{C}=\bigotimes_{i=1}^{r} \mathbf{C}_{\zeta_{i}}
$$

of the above complexes is a bounded complex of projective modules.

Proof. Since the tensor product of any module with a projective module is projective, all the modules in $\mathrm{C}$ except possibly $\bigotimes_{i=1}^{r} P_{n_{i}-1} / L_{\zeta_{i}}=C_{s}, s=$ $\sum_{i=1}^{r}\left(n_{i}-1\right)$, are projective. The latter is at least projective as an $R$-module. By [10, Theorem 78.1], it suffices to prove the theorem in case $R=k$ is a field. Since $k G$ is self-injective, it is enough to demonstrate that $\mathbf{C}$ has a finite projective resolution. So by Proposition 2.6 it suffices to prove that $\operatorname{Ext}_{k G}^{n}(\mathbf{C}, \mathbf{C})=0$ for $n$ sufficiently large.

We start by showing that $\operatorname{Ext}_{k G}^{n}(\mathbf{C}, k)=0$ for $n$ sufficiently large. Consider the spectral sequence

$$
E_{2}^{p q}=\operatorname{Ext}_{k G}^{p}\left(H_{q}(\mathbf{C}), k\right) \Rightarrow \operatorname{Ext}_{k G}^{p+q}(\mathbf{C}, k) .
$$

The map $\mathbf{C} \rightarrow \mathbf{C}_{\zeta_{i}}$ given by using the augmentation $\mathbf{C}_{\zeta_{j}} \rightarrow k$ for $j \neq i$ gives rise to a map of spectral sequences to the above spectral sequence from

$$
E_{2}^{p q}\left(\zeta_{i}\right)=\operatorname{Ext}_{k G}^{p}\left(H_{q}\left(\mathbf{C}_{\zeta_{i}}\right), k\right) \Rightarrow \operatorname{Ext}_{k G}^{p+q}\left(\mathbf{C}_{\zeta_{i}}, k\right) .
$$

The latter spectral sequence was examined in Lemma 3.1, where it was shown that the differential $d_{n_{i}}$ is given by $d_{n_{i}}\left(\alpha . \tilde{\zeta}_{i}\right)=\alpha . \zeta_{i}$. It follows that the same formula holds in the original spectral sequence $E_{j}^{p q}$.

Now $E_{2}^{* *}$ is finitely generated as a module over $E_{2}^{* 0} \cong H^{*}(G, k)$ by basis elements in $E_{2}^{0 *}$ (which is dual to $H_{*}(\mathbf{C})$ ). Since the differentials are $E_{2}^{* 0}$-module homomorphisms, it follows that $E_{\infty}^{* *}$ is also finitely generated as a module over $E_{2}^{* 0}$. The discussion preceding the theorem shows that $\zeta_{1}, \ldots, \zeta_{r}$ act as zero on $E_{\infty}^{* *}$, and so $E_{\infty}^{* *}$ is finitely generated as a module over $H^{*}(G, k) /\left(\zeta_{1}, \ldots, \zeta_{r}\right)$. Since $H^{*}(G, k)$ is finitely generated as a module 
over the subring generated by $\zeta_{1}, \ldots, \zeta_{r}$, the quotient by the ideal generated by $\zeta_{1}, \ldots, \zeta_{r}$ is finite dimensional. It follows that $E_{\infty}^{* *}$ is finite dimensional, and therefore so is $\operatorname{Ext}_{k G}^{*}(\mathbf{C}, k)$.

Finally, the spectral sequence

$$
\operatorname{Ext}_{k G}^{p}\left(\mathbf{C}, H_{-q}(\mathbf{C})\right) \Rightarrow \operatorname{Ext}_{k G}^{p+q}(\mathbf{C}, \mathbf{C})
$$

has as its $E_{2}$ term a finite number of nonzero rows each isomorphic to $\operatorname{Ext}_{k G}^{*}(\mathbf{C}, k)$. Thus the $E_{2}$ term is finite dimensional, and so is $\operatorname{Ext}_{k G}^{*}(\mathbf{C}, \mathbf{C})$. This completes the proof.

More generally, we have the following.

Theorem 4.2. Suppose that $R$ is a field, or the ring of integers in an algebraic number field, or one of its localisations or completions. Suppose that $\mathbf{D}$ is a bounded complex of finitely generated $R G$-modules, and $\zeta_{1}, \ldots, \zeta_{r} \in H^{*}(G, R)$ form an h.s.o.p. for $\operatorname{Ext}_{R G}^{*}(\mathbf{D}, \mathbf{D})$ with $\operatorname{deg}\left(\zeta_{i}\right)=n_{i} \geq 2$. Then letting $\mathbf{C}=$ $\otimes_{i=1}^{r} \mathbf{C}_{\zeta_{i}}$, the complex $\mathbf{C} \otimes \mathbf{D}$ is homotopy equivalent to a bounded complex of finitely generated projective modules. In particular, if $\mathbf{D}=M[n]$ is a module concentrated in a single degree, then $\mathbf{C} \otimes \mathbf{D}$ is a bounded complex of finitely generated projective modules.

Proof. This theorem is proved in the same way as before. That is, we get it by reducing to the case where $R=k$ is a field, and using the spectral sequences

$$
\begin{array}{ccc}
\operatorname{Ext}_{k G}^{p}\left(H_{q}(\mathbf{C}), \mathbf{D}^{*} \otimes \mathbf{D}\right) & \Rightarrow & \operatorname{Ext}_{k G}^{p+q}\left(\mathbf{C}, \mathbf{D}^{*} \otimes \mathbf{D}\right) \\
\| l & & \| l \\
\operatorname{Ext}_{k G}^{p}\left(H_{q}(\mathbf{C}) \otimes \mathbf{D}, \mathbf{D}\right) & \Rightarrow & \operatorname{Ext}_{k G}^{p+q}(\mathbf{C} \otimes \mathbf{D}, \mathbf{D})
\end{array}
$$

and

$$
\begin{array}{ccc}
\operatorname{Ext}_{k G}^{p}\left(\mathbf{C} \otimes \mathbf{D} \otimes \mathbf{D}^{*}, H_{-q}(\mathbf{C})\right) & \Rightarrow & \operatorname{Ext}_{k G}^{p+q}\left(\mathbf{C} \otimes \mathbf{D} \otimes \mathbf{D}^{*}, \mathbf{C}\right) \\
\| l & & \| l \\
\operatorname{Ext}_{k G}^{p}\left(\mathbf{C} \otimes \mathbf{D}, H_{-q}(\mathbf{C}) \otimes \mathbf{D}\right) & \Rightarrow & \operatorname{Ext}_{k G}^{p+q}(\mathbf{C} \otimes \mathbf{D}, \mathbf{C} \otimes \mathbf{D}) .
\end{array}
$$

The following is in a similar vein, and will be used in $\S 10$.

Theorem 4.3. Suppose that $k$ is a field, $\mathbf{D}$ is a bounded complex of finitely generated $k G$-modules, and $\zeta_{1}, \ldots, \zeta_{r}$ is an h.s.o.p. for $H^{*}(G, \mathrm{D})$ with $\operatorname{deg}\left(\zeta_{i}\right)=$ $n_{i} \geq 2$. Then letting $\mathbf{C}=\bigotimes_{i=1}^{r} \mathbf{C}_{\zeta_{i}}$, we have

$$
H^{*}\left(G, L_{1} \otimes \cdots \otimes L_{r} \otimes \mathbf{D}\right)=\operatorname{Ext}_{k G}^{*}(\mathbf{C}, \mathbf{D})=0,
$$

where $L_{i}$ is the cokernel of the injective map $\Omega^{-n_{i}}\left(\hat{\zeta}_{i}\right): k \rightarrow \Omega^{-n_{i}}(k)$.

Proof. It follows as in the last two theorems from the spectral sequence

$$
\operatorname{Ext}_{k G}^{p}\left(H_{q}(\mathbf{C}), \mathbf{D}\right) \Rightarrow \operatorname{Ext}_{k G}^{p+q}(\mathbf{C}, \mathbf{D})
$$

that $\operatorname{Ext}_{k G}^{*}(\mathbf{C}, \mathbf{D})$ is nonzero in only finitely many degrees. Therefore, as in [5] Theorem 1.1, $\operatorname{Ext}_{k G}^{*}(\mathbf{C}, \mathbf{D})=0$. Since $\Omega\left(L_{i}\right)$ is the only nonprojective module in the complex $\mathbf{C}_{\zeta_{i}}^{*}$, and $H^{*}\left(G, \mathbf{C}^{*} \otimes \mathbf{D}\right)=\operatorname{Ext}_{k G}^{*}(\mathbf{C}, \mathbf{D})=0$, it follows that $H^{*}\left(G, L_{1} \otimes \cdots \otimes L_{r} \otimes \mathbf{D}\right)=0$. 


\section{Poincaré duality}

In this section, it is shown that if $\zeta_{1}, \ldots, \zeta_{r}$ is a homogeneous set of parameters for $H^{*}(G, k)$, then $\mathbf{C}_{\zeta_{1}} \otimes \cdots \otimes \mathbf{C}_{\zeta_{r}}$ is homotopy equivalent to its dual, suitably shifted in degree. We use the notation of $\S 3$. However, throughout this section the coefficient ring $R=k$ is a field of characterestic $p$, and hence we can assume that the projective resolution of $k$ as a $k G$-module is minimal. This implies that the kernels $\Omega^{n}(k)$ have no projective submodules.

We begin with a general lemma about homotopy equivalences.

Lemma 5.1. Suppose $\mathbf{C}$ and $\mathbf{D}$ are bounded chain complexes of finitely generated projective $k G$-modules, and $f: \mathbf{C} \rightarrow \mathbf{D}$ is a chain map. Then the following are equivalent.

(i) $f: \mathbf{C} \rightarrow \mathbf{D}$ induces an isomorphism in homology $f_{*}: H_{*}(\mathbf{C}) \stackrel{\cong}{\rightrightarrows} H_{*}(\mathbf{D})$.

(ii) $f$ is a homotopy equivalence.

(iii) There exist decompositions $\mathbf{C}=\mathbf{C}^{\prime} \oplus \mathbf{P}^{\prime}, \mathbf{D}=\mathbf{D}^{\prime} \oplus \mathbf{Q}^{\prime}$, where $\mathbf{P}^{\prime}$ and $\mathbf{Q}^{\prime}$ are exact sequences of projective modules, and the restriction of $f$ to $\mathbf{C}^{\prime}$ is an isomorphism $f: \mathbf{C}^{\prime} \cong \mathbf{D}^{\prime}$.

Proof. It is clear that (iii) $\Rightarrow$ (ii) $\Rightarrow$ (i), so we shall prove that (i) $\Rightarrow$ (iii). Suppose $f: \mathbf{C} \rightarrow \mathbf{D}$ induces an isomorphism in homology. By adding an exact sequence of projective modules $\mathbf{Q}$ to $\mathbf{C}$ we may make $f$ surjective, and still a homology isomorphism. Denote by $\mathbf{P}$ the kernel of $f: \mathbf{C} \oplus \mathbf{Q} \rightarrow \mathbf{D}$. The long exact sequence in homology shows that $\mathbf{P}$ is an exact sequence of projectives. Since projective $k G$-modules are also injective, $\mathbf{P}$ is a bounded exact sequence of injectives, and hence injective as a complex. So the sequence

$$
0 \rightarrow \mathbf{P} \rightarrow \mathbf{C} \oplus \mathbf{Q} \rightarrow \mathbf{D} \rightarrow 0
$$

splits, and $\mathbf{C} \oplus \mathbf{Q} \cong \mathbf{D} \oplus \mathbf{P}$. The result now follows from the Krull-Schmidt theorem for finite chain complexes of finitely generated $k G$-modules.

Now if $\zeta \in H^{n}(G, k)$ is represented by a cocycle $\hat{\zeta}: \Omega^{n} k \rightarrow k$, we may dualise to obtain a map $\hat{\zeta}^{*}: k \rightarrow \Omega^{-n} k$. Applying $\Omega^{n}$, we get a map $\Omega^{n}\left(\hat{\zeta}^{*}\right)$ : $\Omega^{n} k \rightarrow k$. The relationship between this homomorphism and the original map $\hat{\zeta}$ is given in the following proposition.

Proposition 5.2. If $\zeta \in H^{n}(G, k)$ then

$$
\Omega^{n}\left(\hat{\zeta}^{*}\right)=(-1)^{n(n+1) / 2} \hat{\zeta}
$$

Moreover there is a map of chain complexes

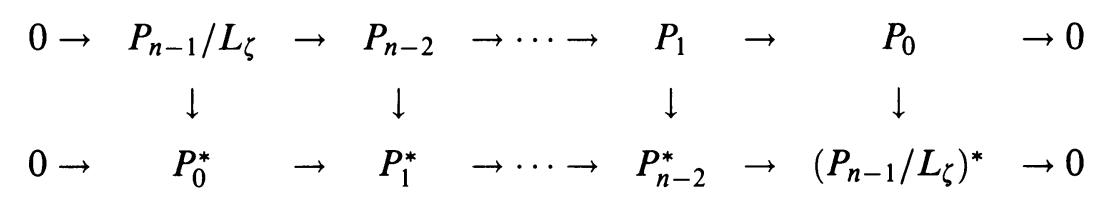

inducing an isomorphism on homology. 
Proof. We build a commutative diagram

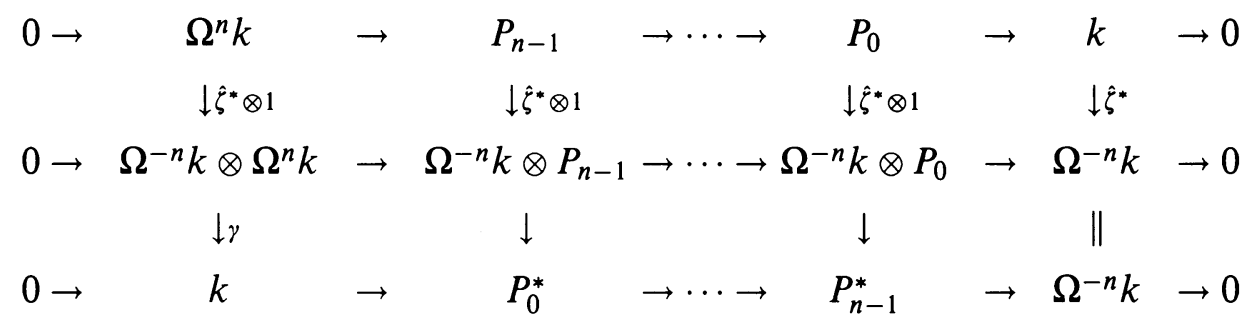

In this diagram, the bottom set of vertical arrows has been filled in using the fact that both rows are exact sequences of projective modules, except at the ends, and $\gamma$ is obtained by restricting the previous map. Now modulo maps which factor through a projective module, there is only one dimension of maps from $\Omega^{-n} k \otimes \Omega^{n} k$ to $k$. It follows that $\gamma$ is some multiple, $\lambda_{n}$.ev, of the evaluation map (regarding $\Omega^{-n} k$ as the dual of $\Omega^{n} k$ ). So the composite of the left-hand vertical maps in the above diagram is $\Omega^{n}\left(\hat{\zeta}^{*}\right)$, and we have

$$
\Omega^{n}\left(\hat{\zeta}^{*}\right)=\lambda_{n} . \mathrm{ev} \circ\left(\hat{\zeta}^{*} \otimes 1\right)=\lambda_{n} . \hat{\zeta} .
$$

(There are no nonzero maps from $\Omega^{n} k$ to $k$ which factor through a projective module.)

It remains to determine the constants $\lambda_{n}$. Since the restriction of $\Omega^{n} k$ to a subgroup $H$ of $G$ is $\Omega^{n} k \oplus$ (projective) and the entire construction commutes with restriction, it suffices to restrict to a cyclic subgroup of $G$ of order $p=$ char $k$ and evaluate $\lambda_{n}$ there. By restricting from $\mathbf{Z} / p \times \mathbf{Z} / p$ to $\mathbf{Z} / p$, it suffices to determine the $\lambda_{n}$ for $\mathbf{Z} / p \times \mathbf{Z} / p$. The cohomology of this group has the property that there are nontrivial products $H^{m} \times H^{n} \rightarrow H^{m+n}$ for every pair of positive integers $m$ and $n$.

Since the map $\zeta \mapsto \lambda_{n} . \zeta$ is an antiautomorphism of $H^{*}(G, k)$ (duality reverses Yoneda composition) we have

$$
\lambda_{m} \xi . \lambda_{n} \zeta=\lambda_{m+n} \zeta . \xi,
$$

so that

$$
\lambda_{m} \lambda_{n}=(-1)^{m n} \lambda_{m+n} .
$$

It remains only to determine $\lambda_{1}$. We leave to the reader the easy exercise of showing that $\lambda_{1}=-1$. Then by induction $\lambda_{n}=(-1)^{n(n+1) / 2}$, as desired.

To prove the second statement of the lemma, we observe that the left-hand map, $\gamma \circ\left(\hat{\zeta}^{*} \otimes 1\right)=(-1)^{n(n+1) / 2} \hat{\zeta}$, in the above diagram has kernel $L_{\zeta}$, while the right-hand map $\hat{\zeta}^{*}$ has cokernel $L_{\zeta}^{*}$, and so by passing to the appropriate quotient on the top row and subcomplex on the bottom row, we obtain a map of complexes

$$
\begin{aligned}
& 0 \rightarrow k \rightarrow P_{n-1} / L_{\zeta} \rightarrow P_{n-2} \rightarrow \cdots \rightarrow P_{1} \rightarrow \quad P_{0} \quad \rightarrow k \rightarrow 0 \\
& (-1)^{n(n+1) / 2} \downarrow \quad \downarrow \quad \downarrow \quad \downarrow \quad \downarrow \quad \downarrow \\
& 0 \rightarrow k \rightarrow P_{0}^{*} \quad \rightarrow \quad P_{1}^{*} \rightarrow \cdots \rightarrow P_{n-2}^{*} \rightarrow\left(P_{n-1} / L_{\zeta}\right)^{*} \rightarrow k \rightarrow 0
\end{aligned}
$$

and hence the required homology equivalence. 
Theorem 5.3. Suppose that $\zeta_{1}, \ldots, \zeta_{r}$ is an h.s.o.p. for $H^{*}(G, k)$ with $\operatorname{deg}\left(\zeta_{i}\right)$ $=n_{i} \geq 2$. Then the complex $\mathbf{C}_{\zeta_{1}} \otimes \cdots \otimes \mathbf{C}_{\zeta_{r}}$ is a direct sum of a complex $\mathbf{C}^{\prime}$ satisfying Poincaré duality in formal dimension $s=\sum_{i=1}^{r}\left(n_{i}-1\right)$,

$$
\operatorname{Hom}_{k}\left(\mathbf{C}^{\prime}, k\right)[s] \cong \mathbf{C}^{\prime}
$$

and an exact complex $\mathbf{P}^{\prime}$ of projective modules.

Proof. By the proposition, we have homology equivalences

$$
\operatorname{Hom}_{k}\left(\mathbf{C}_{\zeta_{i}}, k\right)\left[n_{i}-1\right] \rightarrow \mathbf{C}_{\zeta_{i}} .
$$

Putting these together, we obtain a homology equivalence

$$
\operatorname{Hom}_{k}(\mathbf{C}, k)[s] \cong \mathbf{C} \text {. }
$$

By Theorem 4.1, C is a finite complex of projective modules, and so the theorem now follows by applying Lemma 5.1.

Corollary 5.4. If $S$ is a simple $k G$-module and $\mathbf{C}$ and $s$ are as in the theorem, then the Poincaré series $p_{S}(t)=\sum_{i=0}^{s} t^{i} \operatorname{dim}_{k} \operatorname{Hom}_{k G}\left(C_{i}, S\right)$ satisfies the equation $t^{s} p_{S}(1 / t)=p_{S^{*}}(t)$.

We now summarise what we have established so far about the hypercohomology spectral sequence of the complex $\mathbf{C}$.

Theorem 5.5. Suppose that $\zeta_{1}, \ldots, \zeta_{r}$ is an h.s.o.p. for $H^{*}(G, k)$ with $\operatorname{deg}\left(\zeta_{i}\right)$ $=n_{i} \geq 2$. Then the complex $\mathbf{C}=\mathbf{C}_{\zeta_{1}} \otimes \cdots \otimes \mathbf{C}_{\zeta_{r}}=\mathbf{C}^{\prime} \oplus \mathbf{P}^{\prime}$ is a finite complex of projective modules with cohomology an exterior algebra

$$
H^{*}\left(\operatorname{Hom}_{k}(\mathbf{C}, k)\right)=H^{*}\left(\operatorname{Hom}_{k}\left(\mathbf{C}^{\prime}, k\right)\right)=\Lambda^{*}\left(\tilde{\zeta}_{1}, \ldots, \tilde{\zeta}_{r}\right)
$$

with $\operatorname{deg}\left(\tilde{\zeta}_{i}\right)=n_{i}-1$. The complex $\mathbf{C}^{\prime}$ satisfies Poincaré duality in formal dimension $s=\sum_{i=1}^{r}\left(n_{i}-1\right)$, and $\mathbf{P}^{\prime}$ is a split exact sequence of projectives.

There is a spectral sequence with

$$
E_{2}^{* *}=H^{*}(G, k) \otimes \Lambda^{*}\left(\tilde{\zeta}_{1}, \ldots, \tilde{\zeta}_{r}\right)
$$

and converging to $H^{*}\left(\operatorname{Hom}_{k G}(\mathbf{C}, k)\right)$, which also satisfies Poincaré duality in formal dimension $s$. In this spectral sequence we have $d_{n_{i}}\left(\tilde{\zeta}_{i}\right)=\zeta_{i}$.

If char $k$ is odd, then the above spectral sequence has a multiplicative structure (but see also $§ 3$ for a discussion of the remaining case).

Further information about the differentials in this spectral sequence is given in $\S 11$.

\section{The Cohen-Macaulay CaSe}

We begin with a brief review of Cohen-Macaulay rings.

Definition 6.1. Suppose that $A=\bigoplus_{n>0} A_{n}$ is a finitely generated graded commutative $k$-algebra, and $M=\bigoplus_{n \geq 0} M_{n}$ is a (nonzero) finitely generated graded $A$-module. A sequence $\zeta_{1}, \ldots, \zeta_{r}$ of homogeneous elements of degree $n_{i}>0$ 
in $A$ is said to be a regular sequence for $M$ if for each $i=1, \ldots, r$ the map

$$
M_{n} / M_{n} \cap\left(\zeta_{1}, \ldots, \zeta_{i-1}\right) M \rightarrow M_{n+n_{i}} / M_{n+n_{i}} \cap\left(\zeta_{1}, \ldots, \zeta_{i-1}\right) M
$$

induced by multiplication by $\zeta_{i}$ is injective, for all $n$. Note that we are not asking that $M_{n} / M_{n} \cap\left(\zeta_{1}, \ldots, \zeta_{i-1}\right) M$ be nonzero.

The depth of $M$ is the length of the longest regular sequence. $M$ is said to be Cohen-Macaulay if its depth is equal to its Krull dimension. The ring $A$ is said to be Cohen-Macaulay if it is Cohen-Macaulay as a module over itself. This is equivalent to the condition that there is a polynomial subring $k\left[\zeta_{1}, \ldots, \zeta_{r}\right] \subseteq A$ generated by homogeneous elements $\zeta_{i}$, such that $A$ is a finitely generated free module over $k\left[\zeta_{1}, \ldots, \zeta_{r}\right]$.

The following theorem is proved in Serre [25, p. IV-20, Theorem 2]; see also Stanley [26, Proposition 3.1]. Actually the proof given in Serre [25] is for strictly commutative local rings, but the proof carries over verbatim to the graded commutative case.

Theorem 6.2. Suppose that $A$ is a finitely generated graded commutative $k$-algebra. Then the following are equivalent.

(i) There exists a polynomial subring $k\left[\zeta_{1}, \ldots, \zeta_{r}\right] \subseteq A$ generated by homogeneous elements $\zeta_{i}$, such that $A$ is a finitely generated free module over $k\left[\zeta_{1}, \ldots, \zeta_{r}\right]$.

(ii) For every polynomial subring $k\left[\zeta_{1}, \ldots, \zeta_{r}\right] \subseteq A$ generated by homogeneous elements $\zeta_{i}$, such that $A$ is finitely generated as a module over $k\left[\zeta_{1}, \ldots, \zeta_{r}\right]$ (i.e., $\zeta_{1}, \ldots, \zeta_{r}$ is an h.s.o.p. for $\left.A\right), A$ is a free module.

If $E$ is an elementary abelian $p$-group then $H^{*}(E, k)$ is Cohen-Macaulay. Quillen's calculations [23] show that the cohomology rings of general linear groups at primes other than the natural one are Cohen-Macaulay, and his work on extraspecial 2-groups [22] shows that these also have Cohen-Macaulay cohomology rings. If $G$ is a semidihedral 2-group then $H^{*}(G, k)$ is not Cohen-Macaulay (see Evens and Priddy [14]). Also, if $G$ is a split metacyclic $p$-group with $p$ odd, then usually $H^{*}(G, k)$ is not Cohen-Macaulay (see Diethelm [12]).

It is interesting to note that, until recently (see [1]), in every example which had been computed, $H^{*}(G, k)$ was Cohen-Macaulay whenever $G$ was simple. This is not a general fact because, as noted in the introduction, the CohenMacaulay condition requires that all irreducible components of the maximal ideal spectrum of $H^{*}(G, k)$ have the same dimension. Hence if $G$ is any group having maximal elementary abelian $p$-subgroups of unequal ranks, then $H^{*}(G, k)$ is not Cohen-Macaulay.

Theorem 6.3. Suppose that $H^{*}(G, k)$ is Cohen-Macaulay, and that $\zeta_{1}, \ldots, \zeta_{r}$ is an irredundant h.s.o.p. with $\operatorname{deg}\left(\zeta_{i}\right)=n_{i} \geq 2$. Then the quotient

$$
H^{*}(G, k) /\left(\zeta_{1}, \ldots, \zeta_{r}\right)
$$

satisfies Poincaré duality with formal dimension $s=\sum_{i=1}^{r}\left(n_{i}-1\right)$. Proof. Let $\mathbf{C}=\mathbf{C}_{\zeta_{1}} \otimes \cdots \otimes \mathbf{C}_{\zeta_{r}}=\mathbf{C}^{\prime} \oplus \mathbf{P}^{\prime}$ as in Theorem 5.3. Then by Theorem 5.5 we have a spectral sequence with

$$
E_{2}^{* *}=H^{*}(G, k) \otimes \Lambda^{*}\left(\tilde{\zeta}_{1}, \ldots, \tilde{\zeta}_{r}\right) .
$$


In $\S 3$, we described enough differentials in this spectral sequence to be able to identify it with a staggered version of the Koszul complex for $H^{*}(G, k)$ as a module over $k\left[\zeta_{1}, \ldots, \zeta_{r}\right]$. Since $H^{*}(G, k)$ is Cohen-Macaulay, it is a free module over $k\left[\zeta_{1}, \ldots, \zeta_{r}\right]$, and so the $E_{\infty}$ page of this spectral sequence is the quotient $H^{*}(G, k) /\left(\zeta_{1}, \ldots, \zeta_{r}\right)$ concentrated along the bottom row. It follows that we have an isomorphism

$$
H^{*}(G, k) /\left(\zeta_{1}, \ldots, \zeta_{r}\right) \cong H^{*}\left(\operatorname{Hom}_{k G}(\mathbf{C}, k)\right),
$$

so that this quotient of the cohomology ring satisfies Poincare duality with formal dimension $s=\sum_{i=1}^{r}\left(n_{i}-1\right)$.

Remark 6.4. The proof of this theorem also shows that if $\mathbf{D}$ is a bounded chain complex of $k G$-modules with the property that $H^{*}(G, \mathbf{D})$ is Cohen-Macaulay as a module over $H^{*}(G, k)$, then

$$
H^{*}(G, \mathbf{D}) /\left(\zeta_{1}, \ldots, \zeta_{r}\right) \cong H^{*}\left(\operatorname{Hom}_{k G}(\mathbf{C}, \mathbf{D})\right) \text {. }
$$

Corollary 6.5. Suppose that $H^{*}(G, k)$ is Cohen-Macaulay, and that $\zeta_{1}, \ldots, \zeta_{r}$ is an irredundant h.s.o.p. with $\operatorname{deg}\left(\zeta_{i}\right)=n_{i} \geq 2$. Then the Poincaré series

$$
P_{k}(t)=\sum_{i \geq 0} t^{i} \operatorname{dim}_{k} H^{i}(G, k)
$$

is of the form $p(t) / \prod_{i=1}^{r}\left(1-t^{n_{i}}\right)$, where $p(t)$ is a polynomial satisfying $p(t)=$ $t^{s} p(1 / t)$ where $s=\sum_{i=1}^{r}\left(n_{i}-1\right)$. As rational functions of $t$, we have

$$
P_{k}(1 / t)=(-t)^{r} P_{k}(t)
$$

Proof. Basis elements of the $k$-vector space $H^{*}(G, k) /\left(\zeta_{1}, \ldots, \zeta_{r}\right)$ may be lifted to give free generators of $H^{*}(G, k)$ as a module over $k\left[\zeta_{1}, \ldots, \zeta_{r}\right]$. So the Poincaré series $P_{k}(t)$ is of the form $p(t) / \prod_{i=1}^{r}\left(1-t^{n_{i}}\right)$, where $p(t)$ is the Poincaré series (polynomial) of $H^{*}(G, k) /\left(\zeta_{1}, \ldots, \zeta_{r}\right)$.

By the above theorem, $H^{*}(G, k) /\left(\zeta_{1}, \ldots, \zeta_{r}\right)$ satisfies Poincare duality with formal dimension $s=\sum_{i=1}^{r}\left(n_{i}-1\right)$, and so we have $t^{s} p(1 / t)=p(t)$. This means that

$$
P_{k}\left(\frac{1}{t}\right)=p\left(\frac{1}{t}\right) \cdot \prod_{i=1}^{r} \frac{1}{1-t^{-n_{i}}}=t^{-s} p(t) \cdot \prod_{i=1}^{r} \frac{t^{n_{i}}}{t^{n_{i}}-1}=(-t)^{r} P_{k}(t)
$$

Remark. By a theorem of Quillen [21], the integer in the above theorem, namely the Krull dimension, $r$, of $H^{*}(G, k)$, is equal to the $p$-rank $r_{p}(G)$.

Corollary 6.6. If $H^{*}(G, k)$ is a polynomial ring, then char $k=2$, the generators are in degree one and $G / O(G)$ is an elementary abelian 2-group (here, $O(G)$ denotes the largest odd order normal subgroup of $G)$.

Proof. If $H^{*}(G, k) \cong k\left[\zeta_{1}, \ldots, \zeta_{r}\right]$ with $\operatorname{deg}\left(\zeta_{i}\right)=n_{i}$ then the Poincaré series is

$$
P_{k}(t)=1 / \prod_{i=1}^{r}\left(1-t^{n_{i}}\right)
$$

and so

$$
P_{k}(1 / t)=(-t)^{n_{1}+\cdots+n_{r}} P_{k}(t)
$$


It thus follows from the above theorem that $n_{1}+\cdots+n_{r}=r$, so that each $n_{i}=1$. In odd characteristic, elements of degree one square to zero, so char $k=2$. Moreover, examining $H^{1}(G, k)$, we see that $G$ has an elementary abelian quotient $E$ with the property that inflation $H^{*}(E, k) \rightarrow H^{*}(G, k)$ is an isomorphism, so the kernel has odd order.

We finish this section by showing that for $H^{*}(G, k)$ to be Cohen-Macaulay it is sufficient (but not necessary) for the cohomology of the Sylow $p$-subgroup to be Cohen-Macaulay. The following lemma is well known.

Lemma 6.7. Let $P$ be a Sylow p-subgroup of $G$. Then

$$
\operatorname{res}_{G, P}: H^{*}(G, k) \rightarrow H^{*}(P, k)
$$

is injective, and makes $H^{*}(P, k)$ into a finitely generated $H^{*}(G, k)$-module, which has $H^{*}(G, k)$ as a direct summand.

Proof. This follows from the fact that the transfer map

$$
\operatorname{tr}_{P, G}: H^{*}(P, k) \rightarrow H^{*}(G, k)
$$

satisfies

(i) $\operatorname{res}_{G, P}\left(\operatorname{tr}_{P, G}(x)\right)=|G: P| x$

(ii) $x \cdot \operatorname{tr}_{P, G}(y)=\operatorname{tr}_{P, G}\left(\operatorname{res}_{G, P}(x) \cdot y\right)$

for $x \in H^{*}(G, k)$ and $y \in H^{*}(P, k)$. Finite generation follows from Evens' theorem (cf. Theorem 2.4) and the fact that the Shapiro isomorphism

$$
H^{*}(P, k) \cong H^{*}\left(G, k_{P} \uparrow^{G}\right)
$$

is an isomorphism of $H^{*}(G, k)$-modules, where $k_{P} \uparrow^{G}$ is the permutation module for $G$ on the cosets of $P$.

Proposition 6.8. Let $P$ be a Sylow p-subgroup of $G$. If $H^{*}(P, k)$ is CohenMacaulay then so is $H^{*}(G, k)$.

Proof. We may choose the polynomial subring $k\left[\zeta_{1}, \ldots, \zeta_{r}\right] \subseteq H^{*}(P, k)$ of part (ii) of Theorem 6.2 to lie inside $H^{*}(G, k)$, by the lemma. Thus $H^{*}(G, k)$ is a direct summand of a finitely generated free module over $k\left[\zeta_{1}, \ldots, \zeta_{r}\right]$ and is hence free.

Remark. The converse of this proposition is not true. For example, the Sylow 2-subgroup of $M_{11}$ is a semidihedral group of order 16, whose cohomology in characteristic two is not Cohen-Macaulay (Evens and Priddy [14]), whereas the cohomology of $M_{11}$ in characteristic two is Cohen-Macaulay, see [3].

Corollary 6.9. If a Sylow p-subgroup $P$ of $G$ is abelian then $H^{*}(G, k)$ is Cohen-Macaulay.

\section{THE LAST SURVIVOR}

In this section we prove Theorem 1.3. The idea is that the top degree element in the cohomology of $\operatorname{Hom}_{k G}\left(\bigotimes_{i=1}^{r} \mathbf{C}_{\zeta_{i}}, k\right)$ lifts to a nonzero element of $H^{*}(G, k)$, which we think of as the "last survivor" of the cohomology of this finite complex.

So let $\zeta_{1}, \ldots, \zeta_{r}$ be an irredundant h.s.o.p. for $H^{*}(G, k)$ with $\operatorname{deg}\left(\zeta_{i}\right)=$ $n_{i} \geq 2$, and let $\mathbf{C}=\mathbf{C}_{\zeta_{1}} \otimes \cdots \otimes \mathbf{C}_{\zeta_{r}}$ be the finite complex of projective modules 
discussed in Theorem 5.5. Let $\mathbf{P}$ be a projective resolution of $k$ as a $k G$ module. Since $\mathbf{P}$ is exact and $\mathbf{C}$ consists of projective modules, there is a chain map $\mathbf{C} \rightarrow \mathbf{P}$ inducing an isomorphism in degree zero homology (the degree zero homology of both complexes is $k$ ), and any two such maps are chain homotopic. So if $\mathbf{D}$ is a bounded complex of $k G$-modules, we obtain a well defined map

$$
e: H^{*}\left(\operatorname{Hom}_{k G}(\mathbf{P}, \mathbf{D})\right)=H^{*}(G, \mathbf{D}) \rightarrow H^{*}\left(\operatorname{Hom}_{k G}(\mathbf{C}, \mathbf{D})\right)
$$

which is the horizontal edge homomorphism of the spectral sequence

$$
E_{2}^{p q}=\operatorname{Ext}_{k G}^{p}\left(H_{q}(\mathbf{C}), \mathbf{D}\right) \Rightarrow H^{*}\left(\operatorname{Hom}_{k G}(\mathbf{C}, \mathbf{D})\right)
$$

that was discussed in $\S 4$. The map $e$ commutes with restriction to subgroups.

We saw in $\S 6$ (see Remark 6.4) that if $H^{*}(G, \mathbf{D})$ is Cohen-Macaulay as a module over $H^{*}(G, k)$ then the $E_{\infty}$ page of this spectral sequence consists of the quotient $H^{*}(G, \mathbf{D}) /\left(\zeta_{1}, \ldots, \zeta_{r}\right)$ concentrated along the bottom row, and so the edge map $e$ is surjective in this case.

We may interpret this as follows. Write $\mathbf{C}$ as $\mathbf{C}^{\prime} \oplus \mathbf{P}^{\prime}$, where $\mathbf{P}^{\prime}$ is an exact sequence of projective modules and $\mathbf{C}^{\prime}$ has no summands which are exact sequences of projective modules (so that according to $\S 5, C^{\prime}$ is self-dual). Then for $S$ a simple $k G$-module we have

$$
H^{*}\left(\operatorname{Hom}_{k G}(\mathbf{C}, S)\right) \cong \operatorname{Hom}_{k G}\left(\mathbf{C}^{\prime}, S\right) .
$$

If $\mathbf{P}$ is a minimal resolution of $k$ then

$$
H^{*}(G, S)=\operatorname{Hom}_{k G}(\mathbf{P}, S) .
$$

Thus if $H^{*}(G, S)$ is a Cohen-Macaulay module of Krull dimension $r$ over $H^{*}(G, k)$ for each simple $k G$-module $S$ in the principal block, the edge homomorphisms

$$
\operatorname{Hom}_{k G}(\mathbf{P}, S) \rightarrow \operatorname{Hom}_{k G}\left(\mathbf{C}^{\prime}, S\right)
$$

are all surjective, and so the original map of complexes $\mathbf{C} \rightarrow \mathbf{P}$ is injective. We have thus proved the following embeddability theorem.

Theorem 7.1. Suppose that for each simple $k G$-module $S$ in the principal block of $k G, H^{*}(G, S)$ is a Cohen-Macaulay module of Krull dimension $r=r_{p}(G)$ over $H^{*}(G, k)$. For an irredundant h.s.o.p. $\zeta_{1}, \ldots, \zeta_{r}$ in $H^{*}(G, k)$ with $\operatorname{deg}\left(\zeta_{i}\right)=n_{i} \geq 2$, we express $\mathbf{C}=\mathbf{C}_{\zeta_{1}} \otimes \cdots \otimes \mathbf{C}_{\zeta_{r}}$ as a direct sum $\mathbf{C}^{\prime} \oplus \mathbf{P}^{\prime}$, where $\mathbf{P}^{\prime}$ is an exact sequence of projective modules and $\mathbf{C}^{\prime}$ has no summands which are exact sequences of projective modules. Then there is an embedding of $\mathbf{C}^{\prime}$ in the minimal resolution of $k$ as a $k G$-module, which induces an isomorphism on $\mathrm{H}_{0}$.

Remark. The hypothesis of this theorem fails to hold if $H^{*}(G, S)$ has Krull dimension smaller than that of $H^{*}(G, k)$. For example, if $G=S L(2,4) \cong A_{5}$ and $\operatorname{char}(k)=2$, then the simple modules in the principal block are $k, M$ and $N$, with $\operatorname{dim}_{k}(M)=\operatorname{dim}_{k}(N)=2$ and with projective covers $P_{k}, P_{M}$ and $P_{N}$. If we take $\zeta_{1}$ and $\zeta_{2}$ to be nonzero elements of degrees two and 
three, then $\mathbf{C}^{\prime}$ is as follows

$$
0 \rightarrow P_{k} \rightarrow P_{M} \oplus P_{N} \rightarrow P_{M} \oplus P_{N} \rightarrow P_{k} \rightarrow 0,
$$

whereas the minimal resolution is of the form

$$
\cdots \rightarrow P_{k} \rightarrow P_{M} \oplus P_{N} \rightarrow P_{k} \rightarrow 0 .
$$

All of this can be verified using diagrams [3].

Now suppose that $\zeta_{1}, \ldots, \zeta_{r}$ is an irredundant h.s.o.p. for $H^{*}(G, k)$ with $\operatorname{deg}\left(\zeta_{i}\right)=n_{i} \geq 2$, and let $\mathbf{C}=\mathbf{C}_{\zeta_{1}} \otimes \cdots \otimes \mathbf{C}_{\zeta_{r}}$ as above. By Quillen's theorem [21] on nilpotent elements in $H^{*}(G, k)$, if $E$ is an elementary abelian $p$-subgroup of $G$ of maximal rank, restriction to $E$ is injective on the subring $k\left[\zeta_{1}, \ldots, \zeta_{r}\right]$ of $H^{*}(G, k)$. Since $H^{*}(E, k)$ is Cohen-Macaulay, it follows that if we set $\mathbf{C} \downarrow_{E}=\mathbf{C}^{\prime} \oplus \mathbf{P}^{\prime}$ as above, then $\mathbf{C}^{\prime}$ embeds in the minimal resolution of $k$ as a $k E$-module.

Now in degree $s=\sum_{i=1}^{r}\left(n_{i}-1\right), H^{s}\left(\operatorname{Hom}_{k G}(\mathbf{C}, k)\right)$ is one dimensional, and so we have a canonical map

$$
\gamma: H^{s}(G, k) \stackrel{e}{\rightarrow} H^{s}\left(\operatorname{Hom}_{k G}(\mathbf{C}, k)\right) \stackrel{\cong}{\rightarrow} k .
$$

By Tate duality, this corresponds to an element

$$
\gamma\left(\zeta_{1}, \ldots, \zeta_{r}\right) \in \hat{H}^{-s-1}(G, k) \cong \operatorname{Hom}_{k}\left(H^{s}(G, k), k\right) .
$$

The above discussion shows that

$$
\operatorname{res}_{G, E} \gamma\left(\zeta_{1}, \ldots, \zeta_{r}\right)=\gamma\left(\operatorname{res}_{G, E}\left(\zeta_{1}\right), \ldots, \operatorname{res}_{G, E}\left(\zeta_{r}\right)\right) \neq 0
$$

in $\hat{H}^{-s-1}(E, k)$, and hence $\gamma\left(\zeta_{1}, \ldots, \zeta_{r}\right) \neq 0$ in $\hat{H}^{-s-1}(G, k)$. Since the edge homomorphism $e$, and hence also the map $\gamma$, factors through $H^{*}(G, k) /\left(\zeta_{1}, \ldots, \zeta_{r}\right)$, it follows that there is a nonzero element of degree $s$ in $H^{*}(G, k)$ not in the ideal generated by $\zeta_{1}, \ldots, \zeta_{r}$. Moreover, since the Tate dual of restriction is transfer, this nonzero element may be taken to be a transfer from $H^{*}(E, k)$. This completes the proof of Theorem 1.3.

In fact in the Cohen-Macaulay case, the element $\gamma\left(\zeta_{1}, \ldots, \zeta_{r}\right) \in$ $\hat{H}^{-s-1}(G, k)$ described above determines the ideal $\left(\zeta_{1}, \ldots, \zeta_{r}\right)$ of $H^{*}(G, k)$, as we now prove.

Theorem 7.2. Suppose that $H^{*}(G, k)$ is Cohen-Macaulay, and that $\zeta_{1}, \ldots, \zeta_{r}$ is an irredundant h.s.o.p. Let $\mathbf{C}=\mathbf{C}_{\zeta_{1}} \otimes \cdots \otimes \mathbf{C}_{\zeta_{r}}$. Then

$$
H_{*}\left(\operatorname{Hom}_{k G}(k, \mathbf{C})\right) \cong \gamma\left(\zeta_{1}, \ldots, \zeta_{r}\right) \cap H^{*}(G, k)
$$

(cap product). If $\zeta_{1}^{\prime}, \ldots, \zeta_{r}^{\prime}$ is another irredundant h.s.o.p. with

$$
\gamma\left(\zeta_{1}, \ldots, \zeta_{r}\right)=\gamma\left(\zeta_{1}^{\prime}, \ldots, \zeta_{r}^{\prime}\right)
$$

then setting $\mathbf{D}=\mathbf{C}_{\zeta_{1}^{\prime}} \otimes \cdots \otimes \mathbf{C}_{\zeta_{r}^{\prime}}$, we have isomorphisms

$$
\begin{aligned}
& H_{*}\left(\operatorname{Hom}_{k G}(k, \mathbf{C})\right) \cong H_{*}\left(\operatorname{Hom}_{k G}(k, \mathbf{D})\right), \\
& H^{*}\left(\operatorname{Hom}_{k G}(\mathbf{C}, k)\right) \cong H^{*}\left(\operatorname{Hom}_{k G}(\mathbf{D}, k)\right),
\end{aligned}
$$

and the ideals $\left(\zeta_{1}, \ldots, \zeta_{r}\right)$ and $\left(\zeta_{1}^{\prime}, \ldots, \zeta_{r}^{\prime}\right)$ are equal. 
Proof. First note that $\gamma(\mathbf{C})=\gamma\left(\zeta_{1}, \ldots, \zeta_{r}\right)$ is a class in $\hat{H}^{-s-1}(G, k) \cong$ $H_{s}(G, k)$, and that cap product is the map $H_{s}(G, k) \times H^{t}(G, k) \rightarrow H_{s-t}(G, k)$ for $s \leq t$ induced by cup product in Tate cohomology. Let $\mathbf{P}$ be the minimal projective resolution of $k$ as a $k G$-module. Set $\mathbf{C}=\mathbf{C}^{\prime} \oplus \mathbf{P}^{\prime}$ with $\mathbf{P}^{\prime}$ an exact sequence of projective modules and $\mathbf{C}^{\prime}$ having no summands of this form. Then there is a map $\mathbf{C}^{\prime} \stackrel{\phi}{\rightarrow} \mathbf{P}$ which induces an isomorphism in $H_{0}$. Moreover, by the same argument as used in the proof of Theorem 7.1, $\phi^{*}: \operatorname{Hom}_{k G}(\mathbf{P}, k) \rightarrow$ $\operatorname{Hom}_{k G}\left(\mathbf{C}^{\prime}, k\right)$ is surjective. The dual $\operatorname{Hom}_{k}(\mathbf{P}, k)$ is the minimal injective resolution of $k$ and we have a map $\phi^{*}: \operatorname{Hom}_{k}(\mathbf{P}, k) \rightarrow \operatorname{Hom}_{k}\left(\mathbf{C}^{\prime}, k\right)$ which is a surjection on $G$-fixed points. Let $\psi$ be the composite map

$$
\operatorname{Hom}_{k}(\mathbf{P}, k)[s] \stackrel{\phi^{*}}{\rightarrow} \operatorname{Hom}_{k}\left(\mathbf{C}^{\prime}, k\right)[s] \stackrel{\theta}{\rightarrow} \mathbf{C}^{\prime} \stackrel{\phi}{\hookrightarrow} \mathbf{P}
$$

where $s=\sum_{i=1}^{r}\left(\operatorname{deg}\left(\zeta_{i}\right)-1\right)$ and $\theta$ is the isomorphism given in Theorem 5.3. Now the homology of $\operatorname{Hom}_{k}(\mathbf{P}, k)$ is given by the dual $\varepsilon^{*}$ of the augmentation in degree zero. Now $\psi \circ \varepsilon^{*}$ is a nonzero multiple $a . \gamma(\mathbf{C})$, so the map

$$
\begin{array}{ccc}
H^{t}(G, k) & \longrightarrow & H_{s-t}(G, k) \\
\| l & & \| l \\
H_{-t}\left(\operatorname{Hom}_{k G}(\mathbf{P}, k)\right) & \stackrel{\psi_{*}}{\longrightarrow} & H_{s-t}\left(\operatorname{Hom}_{k G}(k, \mathbf{P})\right)
\end{array}
$$

is cap product with a. $\gamma(\mathbf{C})$. By Theorem 7.1, the map

$$
\phi^{*}: H_{*}\left(\operatorname{Hom}_{k G}(\mathbf{P}, k)\right) \rightarrow H_{*}\left(\operatorname{Hom}_{k G}\left(\mathbf{C}^{\prime}, k\right)\right)
$$

is surjective,

$$
\theta_{*}: H_{*}\left(\operatorname{Hom}_{k G}\left(\mathbf{C}^{\prime}, k\right)\right)[s] \rightarrow H_{*}\left(\operatorname{Hom}_{k G}\left(k, \mathbf{C}^{\prime}\right)\right)=H_{*}\left(\operatorname{Hom}_{k G}(k, \mathbf{C})\right)
$$

is an isomorphism, and

$$
\phi_{*}: H_{*}\left(\operatorname{Hom}_{k G}\left(k, \mathbf{C}^{\prime}\right)\right) \rightarrow H_{*}\left(\operatorname{Hom}_{k G}(k, \mathbf{P})\right)
$$

is injective. So we have proved that

$$
H_{*}\left(\operatorname{Hom}_{k G}(k, \mathbf{C})\right) \cong \gamma\left(\zeta_{1}, \ldots, \zeta_{r}\right) \cap H^{*}(G, k) .
$$

It follows that if $\gamma\left(\zeta_{1}, \ldots, \zeta_{r}\right)$ is equal to $\gamma\left(\zeta_{1}^{\prime}, \ldots, \zeta_{r}^{\prime}\right)$ as an element of $\hat{H}^{-s-1}(G, k)$, then we have an isomorphism

$$
H_{*}\left(\operatorname{Hom}_{k G}(k, \mathbf{C})\right) \cong H_{*}\left(\operatorname{Hom}_{k G}(k, \mathbf{D})\right),
$$

and dually we have an isomorphism $H^{*}\left(\operatorname{Hom}_{k G}(\mathbf{C}, k)\right) \cong H^{*}\left(\operatorname{Hom}_{k G}(\mathbf{D}, k)\right)$ as modules over $H^{*}(G, k)$. The annihilator ideals of these modules are therefore equal. We saw in $\S 6$ that $H^{*}\left(\operatorname{Hom}_{k G}(\mathbf{C}, k)\right) \cong H^{*}(G, k) /\left(\zeta_{1}, \ldots, \zeta_{r}\right)$, so that the annihilator of this module is the ideal $\left(\zeta_{1}, \ldots, \zeta_{r}\right)$.

\section{Filtrations on PRojective Modules}

The aim of this section is to show how a complex $\mathbf{C}$ of $k G$-modules which has at most one nonprojective term $M$ gives rise to a filtration on $M \oplus P$, for some projective module $P$, where the composition factors are Heller translates 
$\Omega^{ \pm n}$ of the homology of $\mathrm{C}$. In the event that $\mathrm{C}$ is a complex of projective modules, this is a filtration of a projective module. The construction given here is a rudimentary form of the equivariant cohomology spectral sequence-without the cohomology. It would be possible to make a construction by embedding the complex in a projective resolution or something similar. This would be equivalent to resolving the homology of $\mathbf{C}$ piece by piece as in [4]. However, the method given here is cleaner and gives a more satisfactory result.

Of course, any filtration on a module leads naturally to a spectral sequence on the cohomology of the module. If the complex $\mathbf{C}$ is one of those constructed in $\S 4$ then the $E_{2}$-term of the spectral sequence corresponding to the filtration is a very familiar object, namely a Koszul complex.

Suppose that

$$
\mathbf{C}: \quad 0 \rightarrow C_{s} \rightarrow \cdots \rightarrow C_{1} \rightarrow C_{0} \rightarrow 0
$$

is a bounded complex of $k G$-modules. Let

$$
\hat{\mathbf{P}}: \quad \cdots \rightarrow P_{1} \stackrel{\partial}{\rightarrow} P_{0} \stackrel{\partial}{\rightarrow} P_{-1} \stackrel{\partial}{\rightarrow} P_{-2} \rightarrow \cdots
$$

be a doubly infinite projective resolution of the trivial $k G$-module $k$. This means that the complex is exact, and that $\partial\left(P_{0}\right) \cong k$. Now consider the double complex $\mathbf{C} \otimes \hat{\mathbf{P}}$. The total complex of $\mathbf{C} \otimes \hat{\mathbf{P}}$ has no homology and hence is a doubly infinite exact sequence. Also since for each $i$ and $j, C_{i}$ and $P_{j}$ are finitely generated, so is $(\mathbf{C} \otimes \hat{\mathbf{P}})_{m}=\bigoplus_{i+j=m} C_{i} \otimes P_{j}$.

For any index $t$ let $(\mathbf{C} \otimes \hat{\mathbf{P}})^{(t)}$ denote the complex

$$
(\mathbf{C} \otimes \hat{\mathbf{P}})^{(t)}: \quad \cdots \rightarrow(\mathbf{C} \otimes \hat{\mathbf{P}})_{t+2} \rightarrow(\mathbf{C} \otimes \hat{\mathbf{P}})_{t+1} \rightarrow(\mathbf{C} \otimes \hat{\mathbf{P}})_{t} \rightarrow 0 .
$$

It is the truncation of the total complex $\mathbf{C} \otimes \hat{\mathbf{P}}$ at the $t$ th term.

Lemma 8.1. (i) For any bounded complex $\mathbf{C}$ of $k G$-modules, the homology

$$
H_{i}\left((\mathbf{C} \otimes \hat{\mathbf{P}})^{(t)}\right)=0
$$

for $i \neq t$.

(ii) If $0 \rightarrow \mathbf{A} \rightarrow \mathbf{B} \rightarrow \mathbf{C} \rightarrow 0$ is a short exact sequence of complexes of $k G$-modules, then

$$
0 \rightarrow(\mathbf{A} \otimes \hat{\mathbf{P}})^{(t)} \rightarrow(\mathbf{B} \otimes \hat{\mathbf{P}})^{(t)} \rightarrow(\mathbf{C} \otimes \hat{\mathbf{P}})^{(t)} \rightarrow 0
$$

is an exact sequence, and we have an exact sequence

$$
0 \rightarrow H_{t}(\mathbf{A} \otimes \hat{\mathbf{P}})^{(t)} \rightarrow H_{t}(\mathbf{B} \otimes \hat{\mathbf{P}})^{(t)} \rightarrow H_{t}(\mathbf{C} \otimes \hat{\mathbf{P}})^{(t)} \rightarrow 0 .
$$

(iii) If $H_{*}(\mathbf{C})=0$ then $H_{t}\left((\mathbf{C} \otimes \hat{\mathbf{P}})^{(t)}\right)$ is projective. Then

(iv) Suppose that $\mathbf{C}$ is concentrated in degree $i$ (that is, $C_{j}=0$ if $j \neq i$ ).

$$
\left.H_{t}\left((\mathbf{C} \otimes \hat{\mathbf{P}})^{(t)}\right)=\Omega^{t-i}\left(C_{i}\right) \oplus \text { (projective }\right) .
$$

Proof. The first statement follows from the fact that $\mathbf{C} \otimes \hat{\mathbf{P}}$ is exact. The second is easily verified because, for any $i$ and $j$,

$$
0 \rightarrow A_{i} \otimes P_{j} \rightarrow B_{i} \otimes P_{j} \rightarrow C_{i} \otimes P_{j} \rightarrow 0
$$


is exact and hence so is

$$
0 \rightarrow \bigoplus_{i+j=m} A_{i} \otimes P_{j} \rightarrow \bigoplus_{i+j=m} B_{i} \otimes P_{j} \rightarrow \bigoplus_{i+j=m} C_{i} \otimes P_{j} \rightarrow 0 .
$$

The last statement of (ii) is a consequence of the long exact sequence on homology. To demonstrate (iii), let

$$
\mathbf{P}: \quad \cdots \rightarrow P_{2} \rightarrow P_{1} \rightarrow P_{0} \rightarrow 0
$$

be the nonnegative part of $\hat{\mathbf{P}}$, and note that $\mathbf{C} \otimes \mathbf{P}$ has no homology. So $\mathbf{C} \otimes \mathbf{P}$ and hence also $\mathbf{C} \otimes \hat{\mathbf{P}}$ are totally split exact sequences of projective modules. Hence $\partial\left((\mathbf{C} \otimes \hat{\mathbf{P}})_{t}\right)=H_{t}\left((\mathbf{C} \otimes \hat{\mathbf{P}})^{(t)}\right)$ is a projective module. To prove (iv) we need only notice that in this case $(\mathbf{C} \otimes \hat{\mathbf{P}})^{(t)}$ is the complex

$$
\cdots \rightarrow C_{i} \otimes P_{t-i+2} \rightarrow C_{i} \otimes P_{t-i+1} \rightarrow C_{i} \otimes P_{t-i} \rightarrow 0,
$$

which is a projective resolution of

$$
\left.C_{i} \otimes \partial\left(P_{t-i}\right) \cong C_{i} \otimes\left(\Omega^{t-i}(k) \oplus(\text { projective })\right) \cong \Omega^{t-i}\left(C_{i}\right) \oplus \text { (projective }\right)
$$

Theorem 8.2. Suppose that

$$
\mathrm{C}: \quad 0 \rightarrow C_{s} \rightarrow C_{s-1} \rightarrow \cdots \rightarrow C_{1} \rightarrow C_{0} \rightarrow 0
$$

is a bounded complex of $k G$-modules. Let $M=C_{t}$ and suppose that $C_{i}$ is projective whenever $i \neq t$. Then there exists a projective module $P$ and $a$ projective resolution $\mathbf{Q}$ of $M \oplus P$ (i.e., $H_{*}(\mathbf{Q})=H_{0}(\mathbf{Q})=M \oplus P$ ) such that $\mathbf{Q}$ has a filtration by complexes

$$
\mathbf{Q}^{(s)} \subseteq \mathbf{Q}^{(s-1)} \subseteq \cdots \subseteq \mathbf{Q}^{(0)}=\mathbf{Q}
$$

with the following properties.

(i) For every $i \geq 0$ and $1 \leq j \leq s, Q_{i}^{(j)}$ (and hence also $Q_{i}^{(j)} / Q_{i}^{(j+1)}$ ) is projective.

(ii) $H_{*}\left(\mathbf{Q}^{(j)} / \mathbf{Q}^{(j+1)}\right)=H_{0}\left(\mathbf{Q}^{(j)} / \mathbf{Q}^{(j+1)}\right) \cong \Omega^{t-j}\left(H_{j}(\mathbf{C})\right) \oplus$ (projective).

(iii) For every $j$ the induced map

$$
H_{0}\left(\mathbf{Q}^{(j)}\right) \rightarrow H_{0}(\mathbf{Q}) \cong M \oplus P
$$

is injective. Denoting its image by $M_{j}$, this gives a filtration

$$
0 \subseteq M_{s} \subseteq \cdots \subseteq M_{1} \subseteq M_{0} \cong M \oplus P
$$

where $M_{j} / M_{j+1} \cong \Omega^{t-j}\left(H_{j}(\mathbf{C})\right) \oplus$ (projective).

Proof. As before, let

$$
\hat{\mathbf{P}}: \quad \cdots \rightarrow P_{1} \stackrel{\partial}{\rightarrow} P_{0} \stackrel{\partial}{\rightarrow} P_{-1} \stackrel{\partial}{\rightarrow} P_{-2} \rightarrow \cdots
$$

be a doubly infinite projective resolution of $k$. We claim that the complex $(\mathbf{C} \otimes \hat{\mathbf{P}})^{(t)}[t]=\mathbf{Q}$ satisfies the conclusion of the theorem. This is the complex $(\mathbf{C} \otimes \hat{\mathbf{P}})^{(t)}$ as in Lemma 8.1 , shifted down in degree by $t$. Clearly $(\mathbf{C} \otimes \hat{\mathbf{P}})^{(t)}$ is a complex of projectives with homology only in degree zero. So we must first show that $\partial\left((\mathbf{C} \otimes \hat{\mathbf{P}})_{t}\right)=M \oplus($ projective $)$. 
Let $\mathbf{A}^{(j)} \hookrightarrow \mathbf{C}$ be the subcomplex such that $A_{i}^{(j)}=C_{i}$ if $i \leq j$ and $A_{i}^{(j)}=0$ if $i>j$. Then we have an exact sequence for $j \leq k$

$$
0 \rightarrow \mathbf{A}^{(j)} \rightarrow \mathbf{A}^{(k)} \rightarrow \mathbf{A}^{(k)} / \mathbf{A}^{(j)} \rightarrow 0
$$

and by part (ii) of the last lemma we have an exact sequence

$$
0 \rightarrow\left(\mathbf{A}^{(j)} \otimes \hat{\mathbf{P}}\right)^{(t)} \rightarrow\left(\mathbf{A}^{(k)} \otimes \hat{\mathbf{P}}\right)^{(t)} \rightarrow\left(\mathbf{A}^{(k)} / \mathbf{A}^{(j)} \otimes \hat{\mathbf{P}}\right)^{(t)} \rightarrow 0 .
$$

If $j=k-1$ then $\mathbf{A}^{(k)} / \mathbf{A}^{(k-1)}$ is concentrated in degree $k$, and so

$$
H_{t}\left(\left(\mathbf{A}^{(k)} / \mathbf{A}^{(k-1)} \otimes \hat{\mathbf{P}}\right)^{(t)}\right)=\Omega^{t-k}\left(C_{k}\right) \oplus \text { (projective). }
$$

If $k=t$, then $\Omega^{t-k}\left(C_{k}\right) \cong M$, while if $k \neq t$ then this homology is projective. Likewise, for $i<j<k$ we have an exact sequence

$0 \rightarrow H_{t}\left(\left(\mathbf{A}^{(j)} / \mathbf{A}^{(i)} \otimes \hat{\mathbf{P}}\right)^{(t)}\right) \rightarrow H_{t}\left(\left(\mathbf{A}^{(k)} / \mathbf{A}^{(i)} \otimes \hat{\mathbf{P}}\right)^{(t)}\right) \rightarrow H_{t}\left(\left(\mathbf{A}^{(k)} / \mathbf{A}^{(j)} \otimes \hat{\mathbf{P}}\right)^{(t)}\right) \rightarrow 0$.

This last sequence must split because one of the two end terms must be projective (hence also injective). The right-hand end is projective if $j \geq t$, while the left-hand end is projective if $j<t$. We conclude that

$$
H_{t}\left((\mathbf{C} \otimes \hat{\mathbf{P}})^{(t)}\right) \cong \bigoplus_{j=1}^{s} H_{t}\left(\left(\mathbf{A}^{(j)} / \mathbf{A}^{(j-1)} \otimes \hat{\mathbf{P}}\right)^{(t)}\right) \cong M \oplus(\text { projective }) .
$$

For each $i$ let $\mathbf{B}^{(i)} \hookrightarrow \mathbf{C}$ be the subcomplex

$$
\mathbf{B}^{(i)}: \quad 0 \rightarrow C_{s} \rightarrow \cdots \rightarrow C_{i+1} \rightarrow \operatorname{Ker} \partial_{i} \rightarrow 0
$$

which is nonzero only in degrees $i$ to $s$. Of course $\mathbf{B}^{(s)}$ is the module $\operatorname{Ker} \partial_{s}$ concentrated in degree $s$. Also $\mathbf{B}^{(0)}=\mathbf{C}$. Now the complex

$$
\mathbf{B}^{(i)} / \mathbf{B}^{(i+1)}: 0 \rightarrow C_{i+1} / \operatorname{Ker} \partial_{i+1} \rightarrow \operatorname{Ker} \partial_{i} \rightarrow 0
$$

has a subcomplex

$$
\mathbf{D}^{(i)}: 0 \rightarrow C_{i+1} / \operatorname{Ker} \partial_{i+1} \cong \partial\left(C_{i+1}\right) \rightarrow 0
$$

and the quotient $\left(\mathbf{B}^{(i)} / \mathbf{B}^{(i+1)}\right) / \mathbf{D}^{(i)}$ is the complex consisting of the one module $H_{i}(\mathbf{C})$ in degree $i$. By Lemma 8.1(iii), $H_{t}\left(\left(\mathbf{D}^{(i)} \otimes \hat{\mathbf{P}}\right)^{(t)}\right)$ is projective. So from Lemma 8.1(ii) we get that

$$
\left.H_{t}\left(\left(\mathbf{B}^{(i)} / \mathbf{B}^{(i+1)} \otimes \hat{\mathbf{P}}\right)^{(t)}\right) \cong \Omega^{t-i}\left(H_{i}(\mathbf{C})\right) \oplus \text { (projective }\right) .
$$

Again by Lemma 8.1 we see that the rest of the homology groups of the complex

$$
\left(\mathbf{B}^{(i)} / \mathbf{B}^{(i+1)} \otimes \hat{\mathbf{P}}\right)^{(t)} \cong\left(\mathbf{B}^{(i)} \otimes \hat{\mathbf{P}}\right)^{(t)} /\left(\mathbf{B}^{(i+1)} \otimes \hat{\mathbf{P}}\right)^{(t)}
$$

are zero.

So let $\mathbf{Q}^{(j)}=\left(\mathbf{B}^{(j)} \otimes \hat{\mathbf{P}}\right)^{(t)}[t] \subseteq \mathbf{Q}$. It is clear that we have a filtration

$$
\mathbf{Q}^{(s)} \subseteq \mathbf{Q}^{(s-1)} \subseteq \cdots \subseteq \mathbf{Q}^{(0)}=\mathbf{Q}
$$

as in statement (i). Statement (ii) is a consequence of the above paragraph. The third condition follows directly from Lemma 8.1(ii). This completes the proof. 
Now suppose that $\zeta_{1}, \ldots, \zeta_{r}$ is an h.s.o.p. for $H^{*}(G, k)$ with $\operatorname{deg}\left(\zeta_{i}\right)=$ $n_{i} \geq 2$. Let $\mathbf{C}_{\zeta_{i}}$ be the complex described in $\S 3$. Then Theorem 8.2(iii) tells us that there exists a projective module $P(i)$ and a filtration of $P_{n_{i}-1} / L_{\zeta_{i}} \oplus P(i)$ of length two (i.e., a short exact sequence)

$$
0 \rightarrow k \rightarrow P_{n_{i}-1} / L_{\zeta_{i}} \oplus P(i) \rightarrow \Omega^{n_{i}-1}(k) \rightarrow 0 .
$$

Here, we have removed the projective summands from the end two terms, at the same time removing summands from $P(i)$, as we may since projective $k G$-modules are also injective.

In this case the sequence (8.3) is obvious because the map $k \rightarrow P_{n_{i}-1} / L_{\zeta_{i}}$ is the injection of $H_{n_{i}-1}\left(\mathbf{C}_{\zeta_{i}}\right)$. Moreover the sequence represents $\zeta_{i}$ as an extension in

$$
\operatorname{Ext}_{k G}^{1}\left(\Omega^{n_{i}-1}(k), k\right) \cong \operatorname{Ext}_{k G}^{n_{i}}(k, k) \cong H^{i}(G, k) .
$$

The filtration on the projective module

$$
P=P_{n_{1}-1} / L_{\zeta_{1}} \otimes \cdots \otimes P_{n_{r}-1} / L_{\zeta_{r}} \oplus \text { (projective) }
$$

(see Theorem 4.1) is the product filtration. It can be written as

$$
\{0\} \subseteq M_{r} \subseteq \cdots \subseteq M_{1} \subseteq M_{0}=P
$$

where $M_{r} \cong k$ and

$$
M_{r-i} / M_{r-i+1} \cong \bigoplus_{\substack{S \subseteq\left\{\zeta_{1}, \ldots, \zeta_{r}\right\} \\|S|=i}} \Omega^{\operatorname{deg}(S)-i}(k) \oplus \text { (projective) }
$$

where $\operatorname{deg}(S)=\sum_{\zeta_{i} \in S} \operatorname{deg}\left(\zeta_{i}\right)$.

As we noted earlier, any filtration on a module gives rise to a spectral sequence on the cohomology of that module. This can be done in either ordinary or Tate cohomology. Let $\hat{\mathbf{P}}$ be the doubly infinite projective resolution of $k$. For any $k G$-module $N$, we have a two-row spectral sequence coming from the filtration (8.3). If we write it as a cohomology spectral sequence and if we shift the degrees appropriately, then the rows on the $E_{0}$ page are given by the formulas

$$
\begin{aligned}
& \hat{E}_{0}^{t, 1}=\operatorname{Hom}_{k G}\left(\hat{P}_{t-n_{i}+2} \otimes k, N\right), \\
& \hat{E}_{0}^{t, 0}=\operatorname{Hom}_{k G}\left(\hat{P}_{t-n_{i}+1} \otimes \Omega^{n_{i}-1}(k), N\right) .
\end{aligned}
$$

The $E_{2}$ page has rows

$$
\begin{aligned}
& \hat{E}_{2}^{t, 1}=\widehat{\operatorname{Ext}}_{k G}^{t-n_{i}+2}(k, N)=\hat{H}^{t-n_{i}+2}(G, N), \\
& \hat{E}_{2}^{t, 0}=\widehat{\operatorname{Ext}}_{k G}^{t-n_{i}+1}\left(\Omega^{n_{i}-1}(k), N\right)=\hat{H}^{t}(G, N) .
\end{aligned}
$$

Then

$$
\begin{array}{cccc}
d_{2}: & \hat{E}_{2}^{t, 1} & \longrightarrow & \hat{E}_{2}^{t+2,0} \\
& \| & & \| \\
& \hat{H}^{t-n_{i}+2}(G, N) & \longrightarrow & \hat{H}^{t+2}(G, N)
\end{array}
$$


is the connecting homomorphism in the long exact sequence associated to (8.3). Specifically it is given by cup product with $\zeta_{i}$. Of course, the spectral sequence converges to $\widehat{\operatorname{Ext}}_{k G}^{*}\left(P_{n_{i}-1} / L_{\zeta_{i}}, N\right)$ suitably shifted.

On the other hand, suppose we consider the filtration given in (8.4). Here the corresponding spectral sequence converges to zero. It has $r+1$ rows. Again if we shift the degrees appropriately then the terms on the $E_{2}$ page are given by

$$
\begin{aligned}
\hat{E}_{2}^{t, i} & =\widehat{\operatorname{Ext}}_{k G}^{t-s^{\prime}+3 i}\left(M_{i} / M_{i-1}, N\right)=\sum \widehat{\operatorname{Ext}}_{k G}^{t-s^{\prime}+3 i}\left(\Omega^{\operatorname{deg}(S)-i}(k), N\right) \\
& =\sum \hat{H}^{t+\operatorname{deg}(S)-s^{\prime}+2 i}(G, N),
\end{aligned}
$$

where the sum is over all subsets $S$ of $\left\{\zeta_{1}, \ldots, \zeta_{r}\right\}$ having exactly $r-i$ elements. Here $s^{\prime}=\sum_{i=1}^{r} \operatorname{deg}\left(\zeta_{i}\right)$. The $d_{2}$ differential is obvious from the product structure, or from the extension classes which connect the terms in the filtration. An easy analysis then shows the following.

Proposition 8.5. In the above spectral sequence the $\hat{E}_{2}$ page is the Koszul complex of $\hat{H}^{*}(G, N)$ as a $k\left[\zeta_{1}, \ldots, \zeta_{r}\right]$-module. More specifically it is isomorphic to $\hat{H}^{*}(G, N) \otimes_{A} \mathscr{K}$, where $\mathscr{K}$ is the Koszul complex of free modules over $A=k\left[\zeta_{1}, \ldots, \zeta_{r}\right]$ which is the usual A-projective resolution of $k$. Thus the $\hat{E}_{3}$ page of the spectral sequence has terms $\operatorname{Tor}_{A}^{*}\left(k, \hat{H}^{*}(G, N)\right)$.

Remark. The Koszul complexes are well documented in the literature. See for example Mac Lane [16]. For another treatment which is both leisurely and considers spectral sequences as above (see [8]). It should be remarked that the spectral sequence in the proposition is identical to the hypercohomology spectral of the complex $\mathbf{B}$ considered in $\S \S 3$ and 4.

\section{ELIMINATING PROJECTIVES FROM COMPLEXES}

If $0 \neq \zeta \in H^{n}(G, k)$, we write $\hat{\zeta}$ for the corresponding map $\Omega^{n} k \rightarrow k$, where $\Omega^{n} k$ is the $n$th kernel in the minimal projective resolution of the trivial $k G$-module $k$. We write $L_{\zeta}$ for the kernel of $\hat{\zeta}$, so that there is a short exact sequence

$$
0 \rightarrow L_{\zeta} \rightarrow \Omega^{n} k \stackrel{\hat{\zeta}}{\rightarrow} k \rightarrow 0 .
$$

We proved in $\S 4$ that if $\zeta_{1}, \ldots, \zeta_{r}$ is an h.s.o.p. for $H^{*}(G, k)$ then $L_{\zeta_{1}} \otimes$ $\cdots \otimes L_{\zeta}$ is projective (and hence also injective). It follows from this fact, that if we tensor together the complexes $0 \rightarrow \Omega^{n_{i}} k \stackrel{\hat{\zeta}_{i}}{\rightarrow} k \rightarrow 0$ and the module $\Omega^{n} k$, and insert the module $L_{\zeta_{1}} \otimes \cdots \otimes L_{\zeta_{r}} \otimes \Omega^{n} k$ to make it exact, we obtain an exact complex each term of which is a sum of modules of the form $\Omega^{s} k$ and projective modules (note that $\Omega^{s} k \otimes \Omega^{t} k \cong \Omega^{s+t} k \oplus$ (projective)). The question is, to what extent may we strip off the projective modules to leave a complex of modules of the form $\Omega^{s} k$ ?

The following questions are an attempt to formalise this, and the main result of this section is that these questions are equivalent. In the next section, we give some conditions under which a positive answer is guaranteed. In particular, a negative answer implies that $r_{p}(G) \geq 3$. 
Question 9.1. Suppose $\zeta_{1}, \ldots, \zeta_{r}$ is an h.s.o.p. for $H^{*}(G, k)$. Given $n \geq 0$, does there exist an exact sequence of the following form?

$$
\mathbf{X}^{(n)}: \quad 0 \rightarrow X_{r} \stackrel{\nu_{r}}{\rightarrow} X_{r-1} \stackrel{\nu_{r-1}}{\rightarrow} \cdots \rightarrow X_{1} \stackrel{\nu_{1}}{\rightarrow} X_{0} \rightarrow 0
$$

where

(i) $X_{i}^{(n)}=X_{i}=\bigoplus_{S \subseteq\left\{\zeta_{1}, \ldots, \zeta_{r}\right\},|S|=i} \tilde{X}_{S}$.

(ii) $\tilde{X}_{S} \cong \Omega^{n+\operatorname{deg}(S)}(k)$ where $\operatorname{deg}(S)=\sum_{\zeta_{i} \in S} \operatorname{deg}\left(\zeta_{i}\right)$.

(iii) If $S^{\prime}=S \cup\left\{\zeta_{j}\right\}$ with $\zeta_{j} \notin S$, then the part of the boundary map $\nu_{\left|S^{\prime}\right|}$ going from $\tilde{X}_{S^{\prime}}$ to $\tilde{X}_{S}$ is $(-1)^{t}$ times a map representing $\zeta_{j}$, where $t=\left|\left\{i \mid \zeta_{i} \in S, i<j\right\}\right|$.

(iv) If $\left|S^{\prime}\right|=|S|+1$ but $S \nsubseteq S^{\prime}$ then the part of the boundary map $\nu_{\left|S^{\prime}\right|}$ going from $\tilde{X}_{S^{\prime}}$ to $\tilde{X}_{S}$ is zero.

Question 9.2. Suppose $\zeta_{1}, \ldots, \zeta_{r}$ is an h.s.o.p. for $H^{*}(G, k)$. Let $M$ be a $k G$-module, $n \geq 0$, and consider the cochain complex

$$
\mathbf{Y}_{(n)}=\mathbf{Y}\left(M ; n ; \zeta_{1}, \ldots, \zeta_{r}\right)
$$

defined as follows.

$$
\mathbf{Y}_{(n)}: \quad 0 \rightarrow Y^{0} \rightarrow Y^{1} \rightarrow \cdots \rightarrow Y^{r-1} \rightarrow Y^{r} \rightarrow 0
$$

where

(i) $Y_{(n)}^{i}=Y^{i}=\bigoplus_{S \subseteq\left\{\zeta_{1}, \ldots, \zeta_{r}\right\},|S|=i} \tilde{Y}^{S}$.

(ii) $\tilde{Y}^{S} \cong H^{n+\operatorname{deg}(S)}(G, M)$ where $\operatorname{deg}(S)=\sum_{\zeta_{i} \in S} \operatorname{deg}\left(\zeta_{i}\right)$.

(iii) If $S^{\prime}=S \cup\left\{\zeta_{j}\right\}$ with $\zeta_{j} \notin S$, then the part of the coboundary map going from $\tilde{Y}^{S}$ to $\tilde{Y}^{S^{\prime}}$ is $(-1)^{t}$ times cup product with $\zeta_{j}$, where $t=\mid\{i \mid$ $\left.\zeta_{i} \in S, i<j\right\} \mid$.

(iv) If $\left|S^{\prime}\right|=|S|+1$ but $S \nsubseteq S^{\prime}$ then the part of the coboundary map going from $\tilde{Y}^{S}$ to $\tilde{Y}^{S^{\prime}}$ is zero.

Is $\mathbf{Y}_{(n)}$ an exact sequence? (It is easy to check that $\mathbf{Y}_{(n)}$ is a cochain complex.)

The complex $\mathbf{Y}_{(n)}$ is actually a singly graded piece of the $\hat{E}_{2}$ page of the spectral sequence considered at the end of the last section. That is, it is the complex

$$
0 \rightarrow \hat{E}_{2}^{n+s^{\prime}-2 r, r} \rightarrow \hat{E}_{2}^{n+s^{\prime}-2 r+2, r-1} \rightarrow \cdots \rightarrow \hat{E}_{2}^{n+s^{\prime}, 0} \rightarrow 0
$$

where the boundary map is $d_{2}$ and $M$ is replaced by $N$. Interestingly, Proposition 8.5 implies that $\mathbf{Y}_{(n)}$ is an exact sequence for all $n$ sufficiently large. This is a consequence of the fact that the $\operatorname{Tor}_{A}^{*}(k, N)$ has finite $k$-dimension. Moreover the question of whether Question 9.2 has an affirmative answer for all $n>0$ is independent of the choice of the set of parameters. See [8] for more details.

Proposition 9.3. Suppose that $\zeta_{1}, \ldots, \zeta_{r}$ is an h.s.o.p. for $H^{*}(G, k)$. Then the following are equivalent.

(i) Question 9.1 has an affirmative answer for the elements $\zeta_{1}, \ldots, \zeta_{r}$ and for all $n \geq 0$. 
(ii) Question 9.2 has an affirmative answer for the elements $\zeta_{1}, \ldots, \zeta_{r}$, all $n \geq 0$ and all simple $k G$-modules $M$.

Proof. We first prove that (i) implies (ii). Assume that for each $n \geq 0$ there is a complex $\mathbf{X}^{(n)}$ having the prescribed properties. Recall that if $M$ is a simple $k G$-module then

$$
\operatorname{Hom}_{k G}\left(\Omega^{m} k, M\right) \cong H^{m}(G, M)
$$

and

$$
\operatorname{Ext}_{k G}^{l}\left(\Omega^{m} k, M\right) \cong \operatorname{Hom}_{k G}\left(\Omega^{m+l} k, M\right) \cong H^{m+l}(G, M) .
$$

Moreover if $\mu: \Omega^{m+l} k \rightarrow \Omega^{m} k$ represents an element $\zeta \in H^{l}(G, k)$ then

$$
\mu^{*}: \operatorname{Hom}_{k G}\left(\Omega^{m} k, M\right) \rightarrow \operatorname{Hom}_{k G}\left(\Omega^{m+l} k, M\right)
$$

is given by cup product with $\zeta$. It follows that the complex $\operatorname{Hom}_{k G}\left(\mathbf{X}^{(n)}, M\right)$ is isomorphic to $\mathbf{Y}_{(n)}=\mathbf{Y}\left(M, n ; \zeta_{1}, \ldots, \zeta_{r}\right)$ by making the obvious identifications. Moreover

$$
\operatorname{Ext}_{k G}^{l}\left(X_{i}^{(n)}, M\right) \cong Y_{(n+l)}^{i} .
$$

So the complex $\operatorname{Hom}_{k G}\left(\mathbf{X}^{(n)}, M\right)$ can be written as

$$
\begin{aligned}
& 0 \rightarrow \operatorname{Hom}_{k G}\left(X_{0}^{(n)}, M\right) \stackrel{\nu^{(n) *}}{\longrightarrow} \operatorname{Hom}_{k G}\left(X_{1}^{(n)}, M\right) \rightarrow \cdots \rightarrow \operatorname{Hom}_{k G}\left(X_{r}^{(n)}, M\right) \rightarrow 0 \\
& \|2 \quad\| l \quad \| l \\
& 0 \rightarrow \quad Y_{(n)}^{0} \quad \longrightarrow \quad Y_{(n)}^{1} \quad \rightarrow \cdots \rightarrow \quad Y_{(n)}^{r} \quad \rightarrow 0 .
\end{aligned}
$$

Since Hom is left exact, $\nu_{1}^{(n) *}$ is injective. Hence

$$
H^{0}\left(\mathbf{Y}_{(n)}\right) \cong H^{0}\left(\operatorname{Hom}_{k G}\left(\mathbf{X}^{(n)}, M\right)\right)=0 .
$$

The short exact sequence

$$
0 \rightarrow \nu_{2}^{(n)}\left(X_{2}^{(n)}\right) \stackrel{i_{1, n}}{\longrightarrow} X_{1}^{(n)} \stackrel{\nu_{1}^{(n)}}{\longrightarrow} X_{0}^{(n)} \rightarrow 0
$$

gives rise to the long exact sequence

$$
\begin{aligned}
0 \rightarrow & \operatorname{Hom}_{k G}\left(X_{0}^{(n)}, M\right) \stackrel{\nu_{1}^{(n) *}}{\longrightarrow} \operatorname{Hom}_{k G}\left(X_{1}^{(n)}, M\right) \stackrel{i_{1, n}^{*}}{\longrightarrow} \operatorname{Hom}_{k G}\left(\nu_{2}^{(n)}\left(X_{2}^{(n)}\right), M\right) \\
& \rightarrow \operatorname{Ext}_{k G}^{1}\left(X_{0}^{(n)}, M\right) \rightarrow \operatorname{Ext}_{k G}^{1}\left(X_{1}^{(n)}, M\right) \rightarrow \cdots .
\end{aligned}
$$

Now for every $j>0$ the map

$$
\begin{array}{ccc}
\operatorname{Ext}_{k G}^{j}\left(X_{0}^{(n)}, M\right) & \stackrel{\nu_{1}^{(n) *}}{\longrightarrow} & \operatorname{Ext}_{k G}^{j}\left(X_{1}^{(n)}, M\right) \\
\| l & & \| l \\
Y_{(n+j)}^{0} & \stackrel{\nu_{1}^{(n+j) *}}{\longrightarrow} & Y_{(n+j)}^{1}
\end{array}
$$

is injective. So in the long exact sequence, all connecting homomorphisms are zero and in particular $i_{1, n}^{*}$ is surjective. We also have a sequence

$$
0 \rightarrow \nu_{3}^{(n)}\left(X_{3}^{(n)}\right) \stackrel{i_{2, n}}{\longrightarrow} X_{2}^{(n)} \stackrel{\varepsilon_{2, n}}{\longrightarrow} \nu_{2}^{(n)}\left(X_{2}^{(n)}\right) \rightarrow 0
$$


and the corresponding long exact sequence is

$$
\begin{aligned}
0 & \rightarrow \operatorname{Hom}_{k G}\left(\nu_{2}^{(n)}\left(X_{2}^{(n)}\right), M\right) \stackrel{\varepsilon_{2, n}^{*}}{\longrightarrow} \operatorname{Hom}_{k G}\left(X_{2}^{(n)}, M\right) \stackrel{i_{2, n}^{*}}{\longrightarrow} \operatorname{Hom}_{k G}\left(\nu_{3}^{(n)}\left(X_{3}^{(n)}\right), M\right) \\
& \rightarrow \operatorname{Ext}_{k G}^{1}\left(\nu_{2}^{(n)}\left(X_{2}^{(n)}\right), M\right) \stackrel{\varepsilon_{2, n}^{*}}{\longrightarrow} \operatorname{Ext}_{k G}^{1}\left(X_{2}^{(n)}, M\right) \rightarrow \cdots .
\end{aligned}
$$

So the map $\varepsilon_{2, n}^{*}$ is injective, and hence the kernel of

$$
\nu_{2}^{(n) *}=\varepsilon_{2, n}^{*} \circ i_{1, n}^{*}: \operatorname{Hom}_{k G}\left(X_{1}^{(n)}, M\right) \rightarrow \operatorname{Hom}_{k G}\left(X_{2}^{(n)}, M\right)
$$

is precisely

$$
\operatorname{Ker}\left(i_{1, n}^{*}\right)=\nu_{1}^{(n) *}\left(\operatorname{Hom}_{k G}\left(X_{0}^{(n)}, M\right)\right)=\nu_{1}^{(n) *}\left(Y_{(n)}^{0}\right) .
$$

Therefore $H^{1}\left(\mathbf{Y}_{(n)}\right)=0$. Notice further that

$$
\operatorname{Ext}_{k G}^{j}\left(\nu_{2}^{(n)}\left(X_{2}^{(n)}\right), M\right) \cong Y_{(n+j)}^{1} / \nu_{1}^{(n+j) *}\left(Y_{(n+j)}^{0}\right) .
$$

So because $H^{1}\left(\mathbf{Y}_{(n+j)}\right)=0$, the map

$$
\begin{array}{ccc}
\operatorname{Ext}_{k G}^{j}\left(\nu_{2}^{(n)}\left(X_{2}^{(n)}\right), M\right) & \stackrel{\varepsilon_{2, n}^{*}}{\longrightarrow} & \operatorname{Ext}_{k G}^{j}\left(X_{2}^{(n)}, M\right) \\
\| l & \| l \\
Y_{(n+j)}^{1} / \nu_{1}^{(n+j) *}\left(Y_{(n+j)}^{0}\right) & \longrightarrow & Y_{(n+j)}^{2}
\end{array}
$$

is injective for all $n, j \geq 0$. Consequently in the long exact sequence (9.4), the connecting homomorphisms are all zero, and so for every $n, j \geq 0$ the map

$$
\operatorname{Ext}_{k G}^{j}\left(X_{2}^{(n)}, M\right) \stackrel{i_{2, n}^{*}}{\longrightarrow} \operatorname{Ext}_{k G}^{j}\left(\nu_{3}^{(n)}\left(X_{3}^{(n)}\right), M\right)
$$

is surjective, so that $\operatorname{Ext}_{k G}^{j}\left(\nu_{3}^{(n)}\left(X_{3}^{(n)}\right), M\right) \cong Y_{(n+j)}^{2} / \nu_{2}^{(n+j)}\left(Y_{(n+j)}^{1}\right)$. Continuing in this way, we see that $\varepsilon_{3, n}^{*}$ is injective and so $H^{2}\left(\mathbf{Y}_{(n)}\right)=0$, and so on. It follows that the homology of $\mathbf{Y}_{(n)}$ is zero and hence $\mathbf{Y}_{(n)}$ is an exact sequence. This completes the proof that (i) implies (ii).

We now give an alternative proof that (i) implies (ii). Let $(\mathbf{P}, \partial)$ be a projective resolution of the trivial $k G$-module $k$. Consider the double complexes $\mathbf{X}^{(n)} \otimes_{k} \mathbf{P}$. Notice that in each such complex the rows

$$
0 \rightarrow X_{r}^{(n)} \otimes P_{i} \rightarrow \cdots \stackrel{\nu \otimes 1}{\longrightarrow} X_{1}^{(n)} \otimes P_{i} \stackrel{\nu \otimes 1}{\longrightarrow} X_{0}^{(n)} \otimes P_{i} \rightarrow 0
$$

are totally split exact sequences of projective modules. So the total complex of the double complex $\operatorname{Hom}_{k G}\left(X_{*}^{(n)} \otimes P_{*}, M\right)$ is an exact sequence and hence has zero homology.

So consider the spectral sequence of the double complex

$$
E_{0}^{p q}=\operatorname{Hom}_{k G}\left(X_{p}^{(n)} \otimes P_{q}, M\right) .
$$

We obtain one such spectral sequence for each value of $n \geq 0$. Taking the boundary with respect to the differential $d_{0}=(1 \otimes \partial)^{*}$, we obtain the $E_{1}$ term

$$
E_{1}^{p q}=\operatorname{Ext}_{k G}^{q}\left(X_{p}^{(n)}, M\right) \cong Y_{(n+q)}^{p} .
$$


This is because $\left(X_{p}^{(n)} \otimes \mathbf{P}, 1 \otimes \partial\right)$ is a projective resolution of $X_{p}^{(n)}$. The isomorphism with $Y_{(n+q)}^{p}$ was discussed earlier. But notice that the map induced from $(\nu \otimes 1)^{*}$ on $E_{1}^{p q}$ is simply the differential on $\mathbf{Y}_{(n+q)}$. So the homology with respect to this differential is

$$
E_{2}^{p q}=H^{p}\left(\mathbf{Y}_{(n+q)}\right) \text {. }
$$

Since this is a first quadrant cohomological spectral sequence, the terms $E_{2}^{0,0}$ and $E_{2}^{1,0}$ live until the $E_{\infty}$ term. By the previous paragraph the spectral sequence converges to zero. So $E_{\infty}^{p q}=0$ for all $p$ and $q$. Therefore $H^{0}\left(\mathbf{Y}_{(n)}\right)=$ $E_{2}^{0,0}=0$ and $H^{1}\left(\mathbf{Y}_{(n)}\right)=E_{2}^{1,0}=0$. Since this happens for every value of $n \geq 0$, we must have $E_{2}^{0, q}=0$ and $E_{2}^{1, q}=0$ for all $q$. But now $E_{2}^{2,0}$ and $E_{2}^{3,0}$ must live until the $E_{\infty}$ term. So again $H^{2}\left(\mathbf{Y}_{(n)}\right)=E_{2}^{2,0}=0$ and $H^{3}\left(\mathbf{Y}_{(n)}\right)=E_{2}^{3,0}=0$ for every $n \geq 0$. Continuing this argument we see that $H^{*}\left(\mathbf{Y}_{(n)}\right)=0$ for all $n \geq 0$.

We now turn our attention to proving that (ii) implies (i). We are given an h.s.o.p. $\zeta_{1}, \ldots, \zeta_{r}$. For each $i=1, \ldots, r$, let $\mathbf{U}^{(i)}$ be the chain complex

$$
\begin{array}{ccccc}
0 \rightarrow & \Omega^{\operatorname{deg}\left(\zeta_{i}\right)}(k) & \stackrel{\hat{\zeta}_{i}}{\rightarrow} & k & \rightarrow 0 \\
\| & & & \| & \\
0 \rightarrow & U_{1}^{(i)} & \rightarrow & U_{0}^{(i)} & \rightarrow 0
\end{array}
$$

having homology $H_{*}\left(\mathbf{U}^{(i)}\right)=H_{1}\left(\mathbf{U}^{(i)}\right)=L_{\zeta_{i}}$. Let $\mathbf{U}=\mathbf{U}^{(1)} \otimes \cdots \otimes \mathbf{U}^{(r)}$, which has homology

$$
H_{*}(\mathbf{U})=H_{n}(\mathbf{U})=L_{\zeta_{1}} \otimes \cdots \otimes L_{\zeta_{r}},
$$

which is a projective $k G$-module (see the first paragraph of this section). The implication (ii) $\Rightarrow$ (i) is a consequence of the following proposition.

Proposition 9.5. Suppose that Question 9.2 has an affirmative answer for the elements $\zeta_{1}, \ldots, \zeta_{r} \in H^{*}(G, k)$ and all simple $k G$-modules $M$. Then for all $n \geq 0$ there exist complexes $\mathbf{X}^{(n)}$ and $\mathbf{Z}^{(n)}$ such that the following hold.

(i) $\mathbf{U} \otimes \boldsymbol{\Omega}^{n} k \cong \mathbf{X}^{(n)} \oplus \mathbf{Z}^{(n)}$.

(ii) $\mathbf{X}^{(n)}$ is exact $\left(H_{*}\left(\mathbf{X}^{(n)}\right)=0\right)$ and no $X_{i}^{(n)}$ has a projective summand.

(iii) $\mathbf{Z}^{(n)}$ is a complex of projective $k G$-modules with $H_{*}\left(\mathbf{Z}^{(n)}\right)=H_{r}\left(\mathbf{Z}^{(n)}\right)=$ $L_{\zeta_{1}} \otimes \cdots \otimes L_{\zeta_{r}} \otimes \Omega^{n} k$.

Proof. First note that we have an exact sequence

$$
0 \rightarrow H_{r}\left(\mathbf{U} \otimes \Omega^{n} k\right) \stackrel{i}{\rightarrow} U_{r} \otimes \Omega^{n} k \rightarrow \cdots \rightarrow U_{0} \otimes \Omega^{n} k \rightarrow 0
$$

where the map $i$ is the inclusion of the homology, and

$$
H_{r}\left(\mathbf{U} \otimes \Omega^{n} k\right)=L_{\zeta_{1}} \otimes \cdots \otimes L_{\zeta_{r}} \otimes \Omega^{n} k,
$$

which is a projective module. If we let $Z^{\prime}$ be the complex with $Z_{r}^{\prime}=$ $H_{r}\left(\mathrm{U} \otimes \Omega^{n} k\right)$, and $Z_{i}^{\prime}=0$ for $i \neq r$, then we have a split injective map of complexes $\mathbf{Z}^{\prime} \rightarrow \mathbf{U} \otimes \Omega^{n} k$. Let $\mathbf{V}^{(n)}$ be the cokernel, so that $\mathbf{U} \otimes \Omega^{n} k \cong \mathbf{V}^{(n)} \oplus \mathbf{Z}^{\prime}$ and $\mathbf{V}^{(n)}$ is exact. The main step in the proof is to show that the complex $\operatorname{Hom}_{k G}\left(\mathbf{V}^{(n)}, M\right)$ is exact for all simple $k G$-modules $M$. 
First we need some notation. If $A$ and $B$ are $k G$-modules, we denote by the expression $P \operatorname{Hom}_{k G}(A, B)$ the subspace of $\operatorname{Hom}_{k G}(A, B)$ consisting of maps which factor through some projective module. Let

$$
\underline{\operatorname{Hom}}_{k G}(A, B)=\operatorname{Hom}_{k G}(A, B) / P \operatorname{Hom}_{k G}(A, B) \text {. }
$$

Of course if $A$ or $B$ is projective then $\underline{\operatorname{Hom}}_{k G}(A, B)=0$. In the event that $A=\Omega^{s} k \oplus$ (projective), we have $\underline{\operatorname{Hom}}_{k G}(A, B)=H^{s}(G, B)$. In particular, for $M$ simple, $\underline{\operatorname{Hom}}_{k G}\left(U_{i}^{(n)}, M\right) \cong Y^{i}\left(M ; n ; \zeta_{1}, \ldots, \zeta_{r}\right)$. So we have an exact sequence

$$
0 \rightarrow \underline{\operatorname{Hom}}_{k G}\left(V_{0}^{(n)}, M\right) \rightarrow \cdots \rightarrow \underline{\operatorname{Hom}}_{k G}\left(V_{r}^{(n)}, M\right) \rightarrow 0
$$

for any $n \geq 0$. Similarly

$$
\operatorname{Ext}_{k G}^{t}\left(V_{i}^{(n)}, M\right) \cong Y^{i}\left(M ; n+t ; \zeta_{1}, \ldots, \zeta_{r}\right) .
$$

So for $M$ simple, the sequence

$$
0 \rightarrow \operatorname{Ext}_{k G}^{t}\left(V_{0}^{(n)}, M\right) \rightarrow \cdots \rightarrow \operatorname{Ext}_{k G}^{t}\left(V_{r}^{(n)}, M\right) \rightarrow 0
$$

is exact, for all $t>0$ and $n \geq 0$.

Now let $(\mathbf{P}, \partial)$ be a projective resolution of $k$ and let $M$ be a simple $k G$-module. Consider the spectral sequence of the double complex

$$
E_{0}^{p q}=\operatorname{Hom}_{k G}\left(V_{p}^{(n)} \otimes P_{q}, M\right) .
$$

As $\mathbf{V}^{(n)}$ is an exact sequence, the homology of the total complex is zero and so the spectral sequence converges to zero. Taking the boundary $(1 \otimes \partial)^{*}$ we get the $E_{1}$ term

$$
E_{1}^{p q}=\operatorname{Ext}_{k G}^{q}\left(V_{p}^{(n)}, M\right)
$$

Now take the boundary induced by $(\nu \otimes 1)^{*}$ to get

$$
E_{2}^{p q}=H^{p}\left(\operatorname{Ext}_{k G}^{q}\left(V_{*}^{(n)}, M\right),(\nu \otimes 1)^{*}\right) .
$$

If $q>0$ then we have already seen that $\operatorname{Ext}_{k G}^{q}\left(V_{*}^{(n)}, M\right) \cong Y_{(n+q)}^{*}$, which is an exact sequence. So $E_{2}^{p q}=0$ for $q>0$. Therefore the spectral sequence collapses onto the first row of the $E_{2}$ page, and all further differentials are zero. Because the spectral sequence converges to zero, we have

$$
H^{i}\left(\operatorname{Hom}_{k G}\left(\mathbf{V}^{(n)}, M\right),(\nu \otimes 1)^{*}\right)=E_{2}^{i, 0}=0 .
$$

So the complex $\operatorname{Hom}_{k G}\left(\mathbf{V}^{(n)}, M\right)$ is exact.

Now notice that $P \operatorname{Hom}_{k G}\left(\mathbf{V}^{(n)}, M\right)$ is a subcomplex of $\operatorname{Hom}_{k G}\left(\mathbf{V}^{(n)}, M\right)$. Moreover, the quotient complex is

$$
\underline{\operatorname{Hom}}_{k G}\left(\mathbf{V}^{(n)}, M\right) \cong \mathbf{Y}_{(n)} .
$$

So we have an exact sequence of complexes

$$
0 \rightarrow P \operatorname{Hom}_{k G}\left(\mathbf{V}^{(n)}, M\right) \rightarrow \operatorname{Hom}_{k G}\left(\mathbf{V}^{(n)}, M\right) \rightarrow \mathbf{Y}_{(n)} \rightarrow 0
$$

in which the last two terms have no cohomology. So by the long exact sequence in cohomology we have $H^{*}\left(P \operatorname{Hom}_{k G}\left(\mathbf{V}^{(n)}, M\right)\right)=0$. 
With this information we proceed to strip the projective modules from the sequence $\mathbf{V}^{(n)}$. Recall that we have an exact sequence

$$
0 \rightarrow V_{r}^{(n)} \stackrel{\nu}{\rightarrow} V_{r-1}^{(n)} \stackrel{\nu}{\rightarrow} \cdots \stackrel{\nu}{\rightarrow} V_{0}^{(n)} \rightarrow 0 .
$$

Let $P_{M}$ be the projective cover of the simple module $M$. Then

$$
\operatorname{dim}_{k} P \operatorname{Hom}_{k G}\left(V_{i}^{(n)}, M\right)
$$

is the number of summands isomorphic to $P_{M}$ in any direct sum decomposition of $V_{i}^{(n)}$ into indecomposable pieces. Let $i$ be the least index such that we have $P \operatorname{Hom}_{k G}\left(V_{i}^{(n)}, M\right) \neq 0$, and let $0 \neq \theta \in P \operatorname{Hom}_{k G}\left(V_{i}^{(n)}, M\right)$. Then $\theta$ extends to a homomorphism $\psi: V_{i}^{(n)} \rightarrow P_{M}$ (i.e., if $\varepsilon: P_{M} \rightarrow M$ then $\varepsilon \circ \psi=\theta$ ). Since $P \operatorname{Hom}_{k G}\left(\mathbf{V}^{(n)}, M\right)$ is exact, we have $\theta \nu \neq 0$. So we have a map of chain complexes

$$
\begin{aligned}
0 \rightarrow V_{r}^{(n)} \rightarrow V_{r-1}^{(n)} \rightarrow \cdots & \rightarrow \begin{array}{c}
V_{i+1}^{(n)} \\
\psi \nu \downarrow
\end{array} \\
0 & \rightarrow V_{i}^{(n)} \rightarrow \cdots \rightarrow V_{0}^{(n)} \rightarrow 0 \\
\downarrow P_{M} & =P_{M} \rightarrow 0
\end{aligned}
$$

Note that $\psi$ and $\psi \nu$ are surjective because $\varepsilon \psi=\theta \neq 0$ and $\varepsilon \psi \nu=\theta \nu \neq 0$. So if $\mathbf{Q}_{M}$ is the complex $\left(0 \rightarrow P_{M} \stackrel{\text { id }}{\rightarrow} P_{M} \rightarrow 0\right)$ concentrated in degrees $i$ and $i+1$, then we have a split short exact sequence of complexes

$$
0 \rightarrow \mathbf{V}^{(n)^{\prime}} \rightarrow \mathbf{V}^{(n)} \rightarrow \mathbf{Q}_{M} \rightarrow 0
$$

So $\mathbf{V}^{(n)} \cong \mathbf{V}^{(n)^{\prime}} \oplus \mathbf{Q}_{M}$. In this way we can eliminate (in pairs) all projective summands from $\mathbf{V}^{(n)}$ and obtain an exact sequence of the prescribed form.

We now show how the questions above have implications for the Poincare series of the cohomology of $G$.

Proposition 9.6. Suppose that $\zeta_{1}, \ldots, \zeta_{r}$ is an h.s.o.p. for $H^{*}(G, k)$ with $\operatorname{deg}\left(\zeta_{i}\right)=n_{i}$, and let $n \geq 0$. Suppose that Question 9.2 has an affirmative answer. Then

$$
\sum_{i=0}^{r}(-1)^{i} \sum_{\substack{S \subseteq\left\{\zeta_{1}, \ldots, \zeta_{r}\right\} \\|S|=i}} \operatorname{dim}_{k} H^{n+\operatorname{deg}(S)}(G, M)=0 .
$$

for all $n \geq 0$ and all simple modules $M$, where $\operatorname{deg}(S)=\sum_{\zeta_{i} \in S} \operatorname{deg}\left(\zeta_{i}\right)$.

Proof. This is an easy consequence of the exactness of $\mathbf{Y}\left(M ; n ; \zeta_{1}, \ldots, \zeta_{r}\right)$.

Now let $P_{M}(t)=\sum_{i \geq 0} t^{i} \operatorname{dim}_{k} H^{i}(G, M)$ be the Poincaré series for $M$. Set $T=\left\{\zeta_{1}, \ldots, \zeta_{r}\right\}$, multiply equation $(9.7)$ by $t^{n+\operatorname{deg}(T)}$ and sum over $n$. We 
get

$$
\begin{aligned}
0 & =\sum_{n=0}^{\infty} \sum_{S \subseteq T}(-1)^{|S|} t^{n+\operatorname{deg}(T)} \operatorname{dim}_{k} H^{n+\operatorname{deg}(S)}(G, M) \\
& =\sum_{n=0}^{\infty} \sum_{S \subseteq T}(-1)^{|S|} t^{\operatorname{deg}(T)-\operatorname{deg}(S)}\left(t^{n+\operatorname{deg}(S)} \operatorname{dim}_{k} H^{n+\operatorname{deg}(S)}(G, M)\right) \\
& =\sum_{S \subseteq T}(-1)^{|S|} t^{\operatorname{deg}(T)-\operatorname{deg}(S)}\left(\sum_{i=\operatorname{deg}(S)}^{\infty} t^{i} \operatorname{dim}_{k} H^{i}(G, M)\right)
\end{aligned}
$$

so that

$$
\begin{aligned}
& \sum_{S \subseteq T}(-1)^{|S|} t^{\operatorname{deg}(T)-\operatorname{deg}(S)} P_{M}(t) \\
& \quad=\sum_{S \subseteq T}(-1)^{|S|} t^{\operatorname{deg}(T)-\operatorname{deg}(S)}\left(\sum_{i=0}^{\operatorname{deg}(S)-1} t^{i} \operatorname{dim}_{k} H^{i}(G, M)\right) .
\end{aligned}
$$

Now we have

$$
\begin{aligned}
\prod_{j=1}^{r}\left(1-t^{n_{j}}\right) & =(-1)^{r} t^{\operatorname{deg}(T)} \prod_{j=1}^{r}\left(1-t^{-n_{j}}\right) \\
& =(-1)^{r} \sum_{S \subseteq T}(-1)^{|S|} t^{\operatorname{deg}(T)-\operatorname{deg}(S)}
\end{aligned}
$$

and so substituting in the previous equation we find that

$$
\prod_{j=1}^{r}\left(1-t^{n_{j}}\right) P_{M}(t)=(-1)^{r} \sum_{S \subseteq T}(-1)^{|S|} t^{\operatorname{deg}(T)-\operatorname{deg}(S)}\left(\sum_{i=0}^{\operatorname{deg}(S)-1} t^{i} \operatorname{dim}_{k} H^{i}(G, M)\right) .
$$

Replacing $S$ by $T \backslash S$ and noting that $(-1)^{r}(-1)^{|S|}=(-1)^{|T \backslash S|}$, this becomes

$$
P_{M}(t)=\frac{\sum_{S \subseteq T}(-1)^{|S|} t^{\operatorname{deg}(S)}\left(\sum_{i=0}^{\operatorname{deg}(T)-\operatorname{deg}(S)-1} t^{i} \operatorname{dim}_{k} H^{i}(G, M)\right)}{\prod_{j=1}^{r}\left(1-t^{n_{j}}\right)} .
$$

Note that the numerator in the above expression is a polynomial.

\section{QUASI-REgULAR SEQUENCES}

There is a notion weaker that that of a regular sequence, which we now introduce, and which is good enough to give a positive answer to the questions posed in $\S 9$.

Definition 10.1. Suppose that $A=\bigoplus_{n \geq 0} A_{n}$ is a finitely generated graded commutative $k$-algebra, and $M=\bigoplus_{n \geq 0} M_{n}$ is a finitely generated graded $A$ module. A sequence $\zeta_{1}, \ldots, \zeta_{r}$ of homogeneous elements of degree $n_{i}$ in $A$ is said to be a quasi-regular sequence for $M$ if for each $i=1, \ldots, r$ the 
map

$$
M_{n} / M_{n} \cap\left(\zeta_{1}, \ldots, \zeta_{i-1}\right) M \rightarrow M_{n+n_{i}} / M_{n+n_{i}} \cap\left(\zeta_{1}, \ldots, \zeta_{i-1}\right) M
$$

induced by multiplication by $\zeta_{i}$ is injective, whenever $n \geq n_{1}+\cdots+n_{i-1}$. In particular, $\zeta_{1}: M_{n} \rightarrow M_{n+n_{1}}$ is injective for all $n \geq 0$.

Serre has investigated a similar definition in which the generators are assumed to be in the same degree. His conclusions are rather different from ours. For further information see the letter from Serre reproduced in the appendix to Guillemin and Sternberg [15]. Note that our terminology has nothing to do with the terminology of quasi-regular sequences used in Matsumura [17].

Proposition 10.2. Suppose that $A=\bigoplus_{n \geq 0} A_{n}$ is a finitely generated graded commutative $k$-algebra, $M=\bigoplus_{n \geq 0} M_{n}$ is a finitely generated graded A-module, and $\zeta_{1}, \ldots, \zeta_{r}$ is a quasi-regular sequence for $M$. For each $n \geq 0$, consider the cochain complex $\mathbf{Y}_{(n)}=\mathbf{Y}\left(M ; n ; \zeta_{1}, \ldots, \zeta_{r}\right)$ defined as follows:

$$
\mathbf{Y}_{(n)}: 0 \rightarrow Y^{0} \rightarrow Y^{1} \rightarrow \cdots \rightarrow Y^{r} \rightarrow 0
$$

where

(i) $Y_{(n)}^{i}=Y^{i}=\bigoplus_{S \subseteq\left\{\zeta_{1}, \ldots, \zeta_{r}\right\},|S|=i} \tilde{Y}^{S}$.

(ii) $\tilde{Y}^{S} \cong M_{n+\operatorname{deg}(S)}$ where $\operatorname{deg}(S)=\sum_{\zeta_{i} \in S} \operatorname{deg}\left(\zeta_{i}\right)$.

(iii) If $S^{\prime}=S \cup\left\{\zeta_{j}\right\}$ with $\zeta_{j} \notin S$, then the part of the coboundary map going from $\tilde{Y}^{S}$ to $\tilde{Y}^{S^{\prime}}$ is $(-1)^{t}$ times multiplication by $\zeta_{j}$, where $t=\mid\left\{i \mid \zeta_{i} \in S, i<\right.$ $j\} \mid$.

(iv) If $\left|S^{\prime}\right|=|S|+1$ but $S \nsubseteq S^{\prime}$ then the part of the coboundary map going from $\tilde{Y}^{S}$ to $\tilde{Y}^{S^{\prime}}$ is zero.

Then $H^{*}(\mathbf{Y})$ is concentrated in degree $r$, and

$$
H^{r}(\mathbf{Y})=M_{n+\operatorname{deg}(T)} / M_{n+\operatorname{deg}(T)} \cap\left(\zeta_{1}, \ldots, \zeta_{r}\right) M,
$$

where $T=\left\{\zeta_{1}, \ldots, \zeta_{r}\right\}$.

Here the complexes $Y_{(n)}$ are the singly graded pieces of the Koszul complex of $M$ as a $k\left[\zeta_{1}, \ldots, \zeta_{r}\right]$-module, as also in Question 9.2.

Proof. We proceed by induction on $r$. Note that if $r=1$ then the complex $\mathbf{Y}$ is simply

$$
0 \rightarrow M_{n} \stackrel{\zeta_{1}}{\rightarrow} M_{n+\operatorname{deg}\left(\zeta_{1}\right)} \rightarrow 0
$$

and because multiplication by $\zeta_{1}$ is injective, the proposition is obvious in this case.

So assume that $r>1$. It is easy to check that $\mathbf{Y}=\mathbf{Y}\left(M ; n ; \zeta_{1}, \ldots, \zeta_{r}\right)$ is a complex. The subcomplex consisting of all the $\tilde{Y}^{S}$ such that $\zeta_{r} \in S$ is

$$
\mathbf{Y}^{\prime}[1] \cong \mathbf{Y}\left(M ; n+\operatorname{deg}\left(\zeta_{r}\right) ; \zeta_{1}, \ldots, \zeta_{r-1}\right)[1]
$$

and the quotient is

$$
\mathbf{Y} / \mathbf{Y}^{\prime}[1] \cong \mathbf{Y}\left(M ; n ; \zeta_{1}, \ldots, \zeta_{r-1}\right) .
$$

By induction, $\mathbf{Y}^{\prime}$ and $\mathbf{Y} / \mathbf{Y}^{\prime}[1]$ satisfy the conclusion of the proposition. Thus the long exact sequence in cohomology of the short exact sequence $0 \rightarrow \mathbf{Y}^{\prime}[1] \rightarrow$ 
$\mathbf{Y} \rightarrow \mathbf{Y} / \mathbf{Y}^{\prime}[1] \rightarrow 0$ of cochain complexes takes the form

$$
0 \rightarrow H^{r-1}(\mathbf{Y}) \rightarrow H^{r-1}\left(\mathbf{Y} / \mathbf{Y}^{\prime}[1]\right) \stackrel{\theta}{\rightarrow} H^{r-1}\left(\mathbf{Y}^{\prime}\right) \rightarrow H^{r}(\mathbf{Y}) \rightarrow 0 .
$$

But $\theta$ is the map

$$
\begin{aligned}
& M_{n+\operatorname{deg}\left(T^{\prime}\right)} / M_{n+\operatorname{deg}\left(T^{\prime}\right)} \cap\left(\zeta_{1}, \ldots, \zeta_{r-1}\right) M \\
& \quad \stackrel{(-1)^{r-1} \zeta_{r}}{\longrightarrow} M_{n+\operatorname{deg}(T)} / M_{n+\operatorname{deg}(T)} \cap\left(\zeta_{1}, \ldots, \zeta_{r-1}\right) M
\end{aligned}
$$

where $T^{\prime}=\left\{\zeta_{1}, \ldots, \zeta_{r-1}\right\}$ and $T=\left\{\zeta_{1}, \ldots, \zeta_{r}\right\}$. This map is injective, according to the definition of a quasi-regular sequence. This completes the proof of the proposition.

We now consider the case in which $A=H^{*}(G, k)$ for $G$ a finite group and $k$ a field of characteristic $p$, and $M=H^{*}(G, \mathbf{D})$ for $\mathbf{D}$ a bounded complex of finitely generated $k G$-modules.

Proposition 10.3. Let $\mathbf{D}$ be a bounded complex of finitely generated $k G$-modules, and suppose that $\zeta_{1}, \ldots, \zeta_{r}$ are nonzero elements of $H^{*}(G, k)$ forming an h.s.o.p. for $H^{*}(G, \mathbf{D})$, with the property that $\zeta_{1}, \ldots, \zeta_{r-1}$ is a quasi-regular sequence for $H^{*}(G, \mathrm{D})$. Then $\zeta_{1}, \ldots, \zeta_{r}$ is a quasi-regular sequence for the module $H^{*}(G, \mathbf{D})$ and the complex $\mathbf{Y}=\mathbf{Y}\left(H^{*}(G, \mathbf{D}) ; n ; \zeta_{1}, \ldots, \zeta_{r}\right)$ is exact (i.e., $H^{*}(\mathbf{Y})=0$ ) for all $n \geq 0$.

Proof. Each $\zeta_{i}$ is represented by a unique nonzero homomorphism $\Omega^{-n_{i}}\left(\hat{\zeta}_{i}\right)$ : $k \rightarrow \Omega^{-n_{i}}(k)$ (cf. Proposition 6.2). Let $L_{i}$ be the cokernel of $\Omega^{-n_{i}}\left(\hat{\zeta}_{i}\right)$, so that we have a short exact sequence

$$
0 \rightarrow k \stackrel{\Omega^{-n_{i}}\left(\hat{\zeta}_{i}\right)}{\longrightarrow} \Omega^{-n_{i}}(k) \rightarrow L_{i} \rightarrow 0 .
$$

Let $\mathbf{V}_{(j, n)}=\mathbf{Y}\left(H^{*}(G, \mathbf{D}) ; n ; \zeta_{1}, \ldots, \zeta_{j}\right)$. We claim that $\mathbf{V}_{(j, n)}$ only has cohomology in degree $j$, and that its cohomology there is

$$
\begin{aligned}
H^{j}\left(\mathbf{V}_{(j, n)}\right) & =H^{n+\sum_{i=1}^{j} n_{i}}(G, \mathbf{D}) / H^{n+\sum_{i=1}^{j} n_{i}}(G, \mathbf{D}) \cap\left(\zeta_{1}, \ldots, \zeta_{j}\right) H^{*}(G, \mathbf{D}) \\
& \cong H^{n}\left(G, \mathbf{D} \otimes L_{1} \otimes \cdots \otimes L_{j}\right) .
\end{aligned}
$$

The proof of this fact follows the scheme of the proof of the last proposition. For $j=1$ we have an exact sequence of chain complexes

$$
0 \rightarrow \mathbf{D} \stackrel{1 \otimes \Omega^{-n_{1}}\left(\hat{\zeta}_{1}\right)}{\longrightarrow} \mathbf{D} \otimes \Omega^{-n_{1}}(k) \longrightarrow \mathbf{D} \otimes L_{1} \rightarrow 0
$$

and hence a long exact sequence

$$
\cdots \rightarrow H^{n}(G, \mathbf{D}) \stackrel{\left(1 \otimes \Omega^{-n_{1}}\left(\hat{\zeta}_{1}\right)\right) *}{\longrightarrow} H^{n}\left(G, \mathbf{D} \otimes \Omega^{-n_{1}}(k)\right) \rightarrow H^{n}\left(G, \mathbf{D} \otimes L_{1}\right) \rightarrow \cdots .
$$

But $H^{n}\left(G, \mathbf{D} \otimes \Omega^{-n_{1}}(k)\right) \cong H^{n+n_{1}}(G, \mathbf{D})$ and the map $\left(1 \otimes \Omega^{-n_{1}}\left(\hat{\zeta}_{1}\right)\right)_{*}$ is cup product with $\zeta_{1}$, which is injective. So all the connecting homomorphisms are zero, and the proposition is proved in this case. 
Now suppose that $1<j<r$. Then we have an exact sequence of chain complexes

$$
\begin{aligned}
0 & \rightarrow \mathbf{D} \otimes L_{1} \otimes \cdots \otimes L_{j-1} \stackrel{1 \otimes \Omega^{-n_{j}}\left(\hat{\xi}_{j}\right)}{\longrightarrow} \mathbf{D} \otimes L_{1} \otimes \cdots \otimes L_{j-1} \otimes \Omega^{-n_{j}}(k) \\
& \rightarrow \mathbf{D} \otimes L_{1} \otimes \cdots \otimes L_{j} \rightarrow 0
\end{aligned}
$$

and the long exact sequence in cohomology takes the form

$$
\begin{aligned}
& \cdots \rightarrow H^{n}\left(G, \mathbf{D} \otimes L_{1} \otimes \cdots \otimes L_{j-1}\right) \stackrel{1 \otimes \Omega^{-n_{j}\left(\hat{\xi}_{j}\right)}}{\underset{\zeta_{j}}{\longrightarrow}} H^{n}\left(G, \mathbf{D} \otimes L_{1} \otimes \cdots \otimes L_{j-1} \otimes \Omega^{-n_{j}}(k)\right) \\
& H^{n+n_{j}}\left(G, \mathbf{D} \otimes L_{1} \otimes \cdots \otimes L_{j-1}\right)
\end{aligned}
$$

and by induction multiplication by $\zeta_{j}$ is injective.

As in the proof of the last proposition, we have an exact sequence of complexes

and so taking cohomology we have

$$
0 \rightarrow \mathbf{V}_{\left(j-1, n+n_{j}\right)}[1] \rightarrow \mathbf{V}_{(j, n)} \rightarrow \mathbf{V}_{(j-1, n)} \rightarrow 0
$$

$$
\begin{aligned}
& \begin{array}{cccc}
0 \rightarrow H^{j-1}\left(\mathbf{V}_{(j-1, n)}\right) & \stackrel{\delta}{\rightarrow} & H^{j-1}\left(\mathbf{V}_{\left(j-1, n+n_{j}\right)}\right) & \rightarrow H^{j}\left(\mathbf{V}_{(j, n)}\right) \rightarrow 0 \\
\| \imath & & \| l &
\end{array} \\
& H^{n}\left(G, \mathbf{D} \otimes L_{1} \otimes \cdots \otimes L_{j-1}\right) \stackrel{\zeta_{j}}{\rightarrow} H^{n+n_{j}}\left(G, \mathbf{D} \otimes L_{1} \otimes \cdots \otimes L_{j-1}\right) .
\end{aligned}
$$

So the connecting homomorphism $\delta$, which is equal to cup product with $\zeta_{j}$, is injective with cokernel $H^{n}\left(G, \mathrm{D} \otimes L_{1} \otimes \cdots \otimes L_{j}\right)$, which proves the claim.

Now consider the case in which $j=r$. Note that by Theorem 4.3, we have $H^{*}\left(G, \mathrm{D} \otimes L_{1} \otimes \cdots \otimes L_{r}\right)=0$. Thus multiplication by $\zeta_{r}$ is an isomorphism from $H^{n}\left(G, \mathbf{D} \otimes L_{1} \otimes \cdots \otimes L_{r-1}\right)$ onto $H^{n+n_{r}}\left(G, \mathbf{D} \otimes L_{1} \otimes \cdots \otimes L_{r-1}\right)$. From the exact sequence of complexes

$$
0 \rightarrow \mathbf{V}_{\left(r-1, n+n_{r}\right)}[1] \rightarrow \mathbf{V}_{(r, n)} \rightarrow \mathbf{V}_{(r-1, n)} \rightarrow 0
$$

we get an exact sequence in cohomology

$$
0 \rightarrow H^{r-1}\left(\mathbf{V}_{(r, n)}\right) \rightarrow H^{r-1}\left(\mathbf{V}_{(r-1, n)}\right) \rightarrow H^{r-1}\left(\mathbf{V}_{\left(r-1, n+n_{r}\right)}\right) \rightarrow H^{r}\left(\mathbf{V}_{(r, n)}\right) \rightarrow 0 .
$$

The middle map is an isomorphism, and so $H^{*}\left(\mathbf{V}_{(r, n)}\right)=0$. Hence

$$
\mathbf{V}_{(r, n)}=\mathbf{Y}\left(H^{*}(G, \mathbf{D}) ; n ; \zeta_{1}, \ldots, \zeta_{r}\right)
$$

is an exact sequence.

Corollary 10.4. Let $G$ be a finite group, $k$ a field of characteristic $p$, and $M$ any $k G$-module. Suppose that $\zeta_{1}, \ldots, \zeta_{r}$ are nonzero elements of $H^{*}(G, k)$ forming a h.s.o.p. for $H^{*}(G, M)$, with the property that $\zeta_{1}, \ldots, \zeta_{r-1}$ is a quasiregular sequence for $H^{*}(G, M)$. Then Question 9.2 has an affirmative answer for the elements $\zeta_{1}, \ldots, \zeta_{r}$ and all $n \geq 0$ for the module $M$.

Proof. This follows immediately from Proposition 10.3 by taking for D the module $M$ concentrated in degree zero.

Corollary 10.5. Suppose that $G$ has p-rank 2. Let $\zeta_{1}, \zeta_{2}$ be a h.s.o.p. for $H^{*}(G, k)$. Then either $\zeta_{1}, \zeta_{2}$ is a quasi-regular sequence for $H^{*}(G, k)$ or $\zeta_{2}$, $\zeta_{1}$ is. 
Proof. By the proposition, it suffices to show that either $\zeta_{1}$ or $\zeta_{2}$ is not a zero divisor in $H^{*}(G, k)$. But this follows from an easy spectral sequence argument.

Proposition 10.6. Let $P$ be a Sylow p-subgroup of $G$. If a sequence of homogeneous elements $\zeta_{1}, \ldots, \zeta_{r} \in H^{*}(G, k) \subseteq H^{*}(P, k)$ is a quasi-regular sequence for $H^{*}(P, k)$, then it is also a quasi-regular sequence for $H^{*}(G, k)$.

Proof. This follows directly from Lemma 6.7 and the definition of quasi-regular sequence.

Remark. In [8] it is shown that Corollary 10.4 has a strong converse. That is, if the answer to Question 9.2 is affirmative for some set of parameters and a particular module $M$, then $H^{*}(G, M)$ has a quasi-regular sequence which is also a system of parameters for $H^{*}(G, M)$ in $H^{*}(G, k)$.

\section{SECONDARY OPERATIONS}

In this section, we determine some more of the differentials in the spectral sequence described in Theorem 5.5. These differentials are given in terms of some secondary operations which we describe in terms of matric Massey products. Accounts of the theory of matric Massey products and their use in determining differentials in spectral sequences may be found in May [18], Ravenel [24, Appendix A1.4], and McCleary [19, §8.3.3].

In case $H^{*}(G, \mathbf{D})$ is not Cohen-Macaulay as a module over $H^{*}(G, k)$, the first example of a possibility for a nontrivial differential not determined by the relations $d_{n_{i}}\left(\tilde{\zeta}_{i}\right)=\zeta_{i}$ is the differential $d_{n_{i}+n_{j}-1}\left(\alpha . \tilde{\zeta}_{i} \tilde{\zeta}_{j}\right)$ in case $\alpha . \zeta_{i}=\alpha . \zeta_{j}=0$.

We suppose for simplicity that the multiplication on cohomology is given by a strictly coassociative diagonal approximation $\Delta: \mathbf{P} \rightarrow \mathbf{P} \otimes \mathbf{P}$. This can be arranged by taking for $\mathbf{P}$ the bar resolution. Note, however, that the diagonal approximation $\Delta$ cannot in general be chosen to be strictly cocommutative, and it is this fact that is responsible for the existence of Steenrod operations. Similarly, the lack of strict coassociativity for the minimal resolution is responsible for the usual Massey triple products.

Suppose that $\mathbf{D}$ is a chain complex of $k G$-modules, bounded above, and $\alpha \in H^{a}(G, \mathbf{D})$ has the property that $\alpha . \zeta_{i}=\alpha . \zeta_{j}=0$. Choose cocycles $u$ representing $\alpha$ and $\eta_{i}$ representing $\zeta_{i}$. Since $\alpha . \zeta_{i}=0$ we have $u . \eta_{i}=d u_{i}$, and similarly $u . \eta_{j}=d u_{j}$, for suitable cochains $u_{i}$ and $u_{j}$. Since

$$
\zeta_{i} \zeta_{j}-(-1)^{n_{i} n_{j}} \zeta_{j} \zeta_{i}=0
$$

we have

$$
\eta_{i} \eta_{j}-(-1)^{n_{i} n_{j}} \eta_{j} \eta_{i}=d \eta_{i j}
$$

To simplify some signs, we shall assume that if $p$ is odd, the $n_{i}$ are even. This will be true in the case we are interested in, namely if the $\zeta_{i}$ generate a polynomial ring, since elements of odd degree square to zero in case $p$ is odd. So we have

and we define

$$
d\left(u_{i} \eta_{j}-u_{j} \eta_{i}-(-1)^{a} u \eta_{i j}\right)=0
$$

$$
\left\langle\alpha \mid \zeta_{i}, \zeta_{j}\right\rangle=\left[u_{i} \eta_{j}-u_{j} \eta_{i}-(-1)^{a} u \eta_{i j}\right] \in H^{a+n_{i}+n_{j}-1}(G, \mathbf{D}) .
$$


This operation may be expressed in terms of matric Massey products as follows:

$$
\left\langle\alpha \mid \zeta_{i}, \zeta_{j}\right\rangle=\left\langle\alpha,\left(\zeta_{i}, \zeta_{j}\right),\left(\begin{array}{c}
\zeta_{j} \\
-\zeta_{i}
\end{array}\right)\right\rangle
$$

Lemma 11.1. In the spectral sequence

$$
E_{2}^{p q}\left(\zeta_{i}, \zeta_{j}\right)=\operatorname{Ext}_{k G}^{p}\left(H_{q}\left(\mathbf{C}_{\zeta_{i}} \otimes \mathbf{C}_{\zeta_{j}}\right), \mathbf{D}\right) \Rightarrow \operatorname{Ext}_{k G}^{p+q}\left(\mathbf{C}_{\zeta_{i}} \otimes \mathbf{C}_{\zeta_{j}}, \mathbf{D}\right)
$$

if $\alpha \in H^{*}(G, \mathbf{D})$ with $\alpha . \zeta_{i}=\alpha . \zeta_{j}=0$, then

$$
d_{n_{i}+n_{j}-1}\left(\alpha . \tilde{\zeta}_{i} \tilde{\zeta}_{j}\right)=\left\langle\alpha \mid \zeta_{i}, \zeta_{j}\right\rangle
$$

Proof. Consider the commutative diagram of chain complexes

$$
\begin{aligned}
& \begin{array}{ccc}
0 & 0 & 0 \\
\downarrow & \downarrow \rightarrow \operatorname{Hom}_{k G}\left(\mathbf{P} \otimes \mathbf{C}_{\zeta_{i}}^{(\infty)} \otimes \mathbf{C}_{\zeta_{j}}^{(\infty)}, \mathbf{D}\right)\left[n_{i}+n_{j}\right] \stackrel{\hat{\xi}_{i}}{\rightarrow} \operatorname{Hom}_{k G}\left(\mathbf{P} \otimes \mathbf{C}_{\zeta_{i}}^{(\infty)} \otimes \mathbf{C}_{\zeta_{j}}^{(\infty)}, \mathbf{D}\right)\left[n_{j}\right] \stackrel{\pi_{i}}{\rightarrow} \operatorname{Hom}_{k G}\left(\mathbf{P} \otimes \mathbf{C}_{\zeta_{i}}^{(\infty)} \otimes \mathbf{C}_{\zeta_{j}}, \mathbf{D}\right)\left[n_{j}\right] \rightarrow 0
\end{array} \\
& \downarrow \xi_{j} \quad \downarrow \xi_{j} \quad \downarrow \xi_{j} \\
& 0 \rightarrow \operatorname{Hom}_{k G}\left(\mathbf{P} \otimes \mathbf{C}_{\zeta_{i}}^{(\infty)} \otimes \mathbf{C}_{\zeta_{j}}^{(\infty)}, \mathbf{D}\right)\left[n_{i}\right] \stackrel{\hat{\xi}_{i}}{\rightarrow} \operatorname{Hom}_{k G}\left(\mathbf{P} \otimes \mathbf{C}_{\xi_{i}}^{(\infty)} \otimes \mathbf{C}_{\xi_{j}}^{(\infty)}, \mathbf{D}\right) \stackrel{\pi_{i}}{\rightarrow} \operatorname{Hom}_{k G}\left(\mathbf{P} \otimes \mathbf{C}_{\zeta_{j}}^{(\infty)} \otimes \mathbf{C}_{\zeta_{j}}, \mathbf{D}\right) \rightarrow 0 \\
& \downarrow \pi_{j} \\
& 0 \rightarrow \quad \operatorname{Hom}_{k G}\left(\mathbf{P} \otimes \mathbf{C}_{\zeta_{i}} \otimes \mathbf{C}_{\zeta_{j}}^{(\infty)}, \mathbf{D}\right)\left[n_{i}\right] \stackrel{\hat{\xi}_{i}}{\rightarrow} \quad \operatorname{Hom}_{k G}\left(\mathbf{P} \otimes \mathbf{C}_{\zeta_{i}} \otimes \mathbf{C}_{\zeta_{j}}^{(\infty)}, \mathbf{D}\right) \stackrel{\pi_{i}}{\rightarrow} \quad \operatorname{Hom}_{k G}\left(\mathbf{P} \otimes \mathbf{C}_{\zeta_{i}} \otimes \mathbf{C}_{\zeta_{j}}, \mathbf{D}\right) \quad \rightarrow 0 \\
& \downarrow \\
& 0 \\
& \begin{array}{ll}
\downarrow & \downarrow \\
0 & 0
\end{array}
\end{aligned}
$$

Taking homology, we obtain a mesh of long exact sequences, part of which is as follows:

$$
\begin{aligned}
& \rightarrow \operatorname{Ext}_{k G}^{n_{i}+a-1}(k, \mathbf{D}) \stackrel{\left(\pi_{i}\right)_{*}}{\longrightarrow} \operatorname{Ext}_{k G}^{n_{i}+a-1}\left(\mathbf{C}_{\zeta_{i}}, \mathbf{D}\right) \rightarrow \operatorname{Ext}_{k G}^{a}(k, \mathbf{D}) \stackrel{\zeta_{i}}{\rightarrow} \operatorname{Ext}_{k G}^{n_{i}+a}(k, \mathbf{D}) \\
& \downarrow \zeta_{j} \quad \downarrow \zeta_{j} \quad \downarrow \zeta_{j} \\
& \rightarrow \operatorname{Ext}_{k G}^{n_{i}+n_{j}+a-1}(k, \mathbf{D}) \stackrel{\left(\pi_{i}\right)_{*}}{\longrightarrow} \operatorname{Ext}_{k G}^{n_{i}+n_{j}+a-1}\left(\mathbf{C}_{\zeta_{i}}, \mathbf{D}\right) \rightarrow \operatorname{Ext}_{k G}^{n_{j}+a}(k, \mathbf{D}) \rightarrow
\end{aligned}
$$

The differential

$$
d_{n_{i}+n_{j}-1}: \operatorname{Ext}_{k G}^{a}(k, \mathbf{D}) \rightarrow \operatorname{Ext}_{k G}^{n_{i}+n_{j}+a-1}(k, \mathbf{D})
$$

is the switchback map in this diagram. By Lemma 3.1, the maps marked $\zeta_{i}$, $\zeta_{j}$ are given by multiplication by $\zeta_{i}, \zeta_{j}$. By the definition of the connecting homomorphism in cohomology, $\left[\pi_{i} u_{i}\right] \in \operatorname{Ext}_{k G}^{n_{i}+a-1}\left(\mathbf{C}_{\zeta_{i}}, \mathbf{D}\right)$ has image $\alpha=[u]$ in $\operatorname{Ext}_{k G}^{a}(k, \mathbf{D})$. We have

$$
\begin{aligned}
\left(\pi_{i}\right)_{*}\left\langle\alpha \mid \zeta_{i}, \zeta_{j}\right\rangle & =\left[\pi_{i}\left(u_{i} \eta_{j}-u_{j} \eta_{i}-(-1)^{a} u \eta_{i j}\right)\right] \\
& =\left[\pi_{i} u_{i} \eta_{j}-\left(\pi_{i} \circ \hat{\zeta}_{i}\right) u_{j}\right]=\left[\pi_{i} u_{i}\right] \cdot \zeta_{j}
\end{aligned}
$$

in $\operatorname{Ext}_{k G}^{n_{i}+n_{j}+a-1}\left(\mathbf{C}_{\zeta_{i}}, \mathbf{D}\right)$, and so $\left\langle\alpha \mid \zeta_{i}, \zeta_{j}\right\rangle$ is the image of $u$ under this switchback map. Note that only one of the terms in the expression for $\left\langle\alpha \mid \zeta_{i}, \zeta_{j}\right\rangle$ survives in this calculation; the rest of the terms are to ensure that it is a cocycle. 
The next example of a nontrivial differential is given as follows. If $\alpha \in$ $H^{a}(G, \mathbf{D})$ satisfies $\left\langle\alpha \mid \zeta_{i}, \zeta_{j}\right\rangle=\left\langle\alpha \mid \zeta_{i}, \zeta_{k}\right\rangle=\left\langle\alpha \mid \zeta_{j}, \zeta_{k}\right\rangle=0$, then there is a possibility for a nontrivial differential $d_{n_{i}+n_{j}+n_{k}-2}\left(\alpha . \tilde{\zeta}_{i} \tilde{\zeta}_{j} \tilde{\zeta}_{k}\right)$. Since $\left\langle\alpha \mid \zeta_{i}, \zeta_{j}\right\rangle=$ 0 , we may choose a cochain $u_{i j}$ with

$$
d u_{i j}=u_{i} \eta_{j}-u_{j} \eta_{i}-(-1)^{a} u \eta_{i j}
$$

Now the element

$$
\eta_{i j} \eta_{k}-\eta_{i k} \eta_{j}+\eta_{j k} \eta_{i}-\eta_{i} \eta_{j k}+\eta_{j} \eta_{i k}-\eta_{k} \eta_{i j}
$$

is a coboundary $d \eta_{i j k}$. To see this, note that the complex

$$
\mathrm{Hom}_{k G}(\mathbf{P} \otimes \mathbf{P} \otimes \mathbf{P}, \mathbf{P})
$$

is exact in positive degrees (the negative homology in degree $-n$ is $\operatorname{Ext}_{k G}^{n}(k, k)$ $\left.=H^{n}(G, k)\right)$, and that the map sending $\eta_{i} \otimes \eta_{j} \otimes \eta_{k}$ to the above element is a map of degree +1 whose coboundary is zero.

The cochain

$$
u_{i j} \eta_{k}-u_{i k} \eta_{j}+u_{j k} \eta_{i}-u_{i} \eta_{j k}+u_{j} \eta_{i k}-u_{k} \eta_{i j}-(-1)^{a} u \eta_{i j k}
$$

is a cocycle, and we denote by $\left\langle\alpha \mid \zeta_{i}, \zeta_{j}, \zeta_{k}\right\rangle$ the cohomology class it represents.

In terms of matric Massey products, this is given as follows:

$$
\left\langle\alpha \mid \zeta_{i}, \zeta_{j}, \zeta_{k}\right\rangle=\left\langle\alpha,\left(\zeta_{i}, \zeta_{j}, \zeta_{k}\right),\left(\begin{array}{rrr}
0 & -\zeta_{k} & \zeta_{j} \\
\zeta_{k} & 0 & -\zeta_{i} \\
-\zeta_{j} & \zeta_{i} & 0
\end{array}\right),\left(\begin{array}{l}
\zeta_{i} \\
\zeta_{j} \\
\zeta_{k}
\end{array}\right)\right\rangle
$$

The proof of the following is similar to the proof of Lemma 11.1.

Lemma 11.2. In the spectral sequence

$$
\begin{gathered}
E_{2}^{p q}\left(\zeta_{i}, \zeta_{j}, \zeta_{k}\right)=\operatorname{Ext}_{k G}^{p}\left(H_{q}\left(\mathbf{C}_{\zeta_{i}} \otimes \mathbf{C}_{\zeta_{j}} \otimes \mathbf{C}_{\zeta_{k}}\right), \mathbf{D}\right) \Rightarrow \operatorname{Ext}_{k G}^{p+q}\left(\mathbf{C}_{\zeta_{i}} \otimes \mathbf{C}_{\zeta_{j}} \otimes \mathbf{C}_{\zeta_{k}}, \mathbf{D}\right) \\
\text { if } \alpha \in H^{*}(G, \mathbf{D}) \text { with }\left\langle\alpha \mid \zeta_{i}, \zeta_{j}\right\rangle=\left\langle\alpha \mid \zeta_{i}, \zeta_{k}\right\rangle=\left\langle\alpha \mid \zeta_{j}, \zeta_{k}\right\rangle=0, \text { then } \\
d_{n_{i}+n_{j}+n_{k}-2}\left(\alpha \cdot \tilde{\zeta}_{i} \tilde{\zeta}_{j} \tilde{\zeta}_{k}\right)=\left\langle\alpha \mid \zeta_{i}, \zeta_{j}, \zeta_{k}\right\rangle .
\end{gathered}
$$

The general case of the above construction goes as follows. We define operations $\left\langle\alpha \mid \zeta_{i_{1}}, \ldots, \zeta_{i_{t}}\right\rangle$ under the conditions that $\alpha \zeta_{i_{1}}=\cdots=\alpha \zeta_{i_{t}}=0$, and all operations $\langle\alpha \mid \cdots\rangle$ are zero for $\cdots$ a proper subsequence of $\zeta_{i_{1}}, \ldots, \zeta_{i_{t}}$. The operation is described as a matric Massey product $\left\langle\alpha, M_{1}, \ldots, M_{t}\right\rangle$ where $M_{1}, \ldots, M_{t}$ are matrices whose entries are either zero or plus or minus one of the $\zeta_{i_{j}}$. The rows and columns of $M_{s}(1 \leq s \leq t)$ are indexed by subsets $I$ and $J$ of $\left\{i_{1}, \ldots, i_{t}\right\}$ of sizes $s-1$ and $s$ respectively. If $I \nsubseteq J$ then the corresponding entry is zero, while if $J=I \cup\left\{i_{j}\right\}$ then the corresponding entry is plus or minus $\zeta_{i_{j}}$. The sign is $(-1)$ to the power of the number of elements of $J$ which come before $i_{j}$. It is easy to check that $M_{s} M_{s+1}=0$. Since the chain complex $\operatorname{Hom}_{k G}\left(\mathbf{P}^{\otimes t}, \mathbf{P}\right)$ is exact in positive degrees, the matric Massey products of consecutive elements of the list $M_{1}, \ldots, M_{t}$ vanish. So by the conditions on $\alpha$, the matric Massey product $\left\langle\alpha, M_{1}, \ldots, M_{t}\right\rangle$ is defined, and we write $\left\langle\alpha \mid \zeta_{i_{1}}, \ldots, \zeta_{i_{t}}\right\rangle$ for this expression. An argument similar to the proof 
of Lemma 11.1 shows that if $\alpha . \tilde{\zeta}_{i_{1}} \ldots \tilde{\zeta}_{i_{t}}$ lives to $E_{n_{i_{1}}+\cdots+n_{i_{t}}-t+1}^{* *}$ then

$$
d_{n_{i_{1}}+\cdots+n_{i_{t}}-t+1}\left(\alpha . \tilde{\zeta}_{i_{1}} \ldots \tilde{\zeta}_{i_{t}}\right)=\left\langle\alpha \mid \zeta_{i_{1}}, \ldots, \zeta_{i_{t}}\right\rangle .
$$

An example involving the last survivor described in $\S 7$ is as follows. We look at the spectral sequence

$$
\hat{E}_{2}^{p q}=\widehat{\operatorname{Ext}}_{k G}^{p}\left(H_{q}(\mathbf{C}), k\right) \Rightarrow 0
$$

of the double complex $\operatorname{Hom}_{k G}(\hat{\mathbf{P}} \otimes \mathbf{C}, k)$, where $\hat{\mathbf{P}}$ is a complete resolution of the trivial module. Let $\alpha$ be a nonzero element of the one dimensional space $\hat{H}^{-1}(G, k)$. Then $\alpha . \tilde{\zeta}_{1} \ldots \tilde{\zeta}_{r}\left(r=r_{p}(G)\right)$ transgresses to a nonzero element in the base $\hat{E}_{s, 0}^{s}\left(s=\sum_{i=1}^{r} n_{i}\right)$ of this spectral sequence, representing the last survivor:

$$
\left\langle\alpha \mid \zeta_{1}, \ldots, \zeta_{r}\right\rangle \in E_{\infty}^{s, 0} \cong H^{s}(G, k) / \operatorname{Ker}(e)
$$

A nontrivial example involving elements of positive degree is described in [6].

\section{REFERENCES}

1. A. Adem and J. Milgram, $A_{5}$ invariants and the cohomology of $L_{3}(4)$, Proc. London Math. Soc. (3) 66 (1993), 187-224.

2. G. S. Avrunin and L. L. Scott, Quillen stratification for modules, Invent. Math. 66 (1982), 277-286.

3. D. J. Benson and J. F. Carlson. Diagrammatic methods for modular representations and cohomology, Comm. Algebra 15 (1987), 53-121.

4. __ Complexity and multiple complexes, Math. Z. 195 (1987), 221-238.

5. D. J. Benson, J. F. Carlson, and G. R. Robinson, On the vanishing of group cohomology, J. Algebra 131 (1990), 40-73.

6. D. J. Benson and J. F. Carlson, Products in negative cohomology, J. Pure Appl. Algebra 82 (1992), 107-129.

7. J. F. Carlson. Products and projective resolutions, Proc. Sympos. Pure Math., vol. 47, part 1, Amer. Math. Soc., Providence, RI, 1987, pp. 399-408.

8. _ Projective resolutions and degree shifting for cohomology and group rings, Representations of Algebras and Related Topics (H. Tachikawa and S. Brenner, eds.), London Math. Soc. Lecture Note Series No. 168, Cambridge Univ. Press, Cambridge, 1992, pp. 80-126.

9. H. Cartan and S. Eilenberg, Homological algebra, Princeton Univ. Press, Princeton, NJ, 1956.

10. C. W. Curtis and I. Reiner, Representation theory of finite groups and associative algebras, Wiley-Interscience, New York, 1962.

11. P. Deligne, Séminaire de géométrie algébrique du Bois-Marie $S G A 4 \frac{1}{2}$, Lecture Notes in Math., vol. 569, Springer-Verlag, Berlin and New York, 1977.

12. T. Diethelm, The Modp cohomology rings of the nonabelian split metacyclic p-groups, Arch. Math. 44 (1985), 29-38.

13. L. Evens, The cohomology ring of a finite group, Trans. Amer. Math. Soc. 101 (1961), 224239.

14. L. Evens and S. Priddy, The cohomology of the semi-dihedral group, Conf. Algebraic Topology in Honour of Peter Hilton (R. Piccinini and D. Sjerve, eds.), Contemp. Math., vol. 37, Amer. Math. Soc., Providence, RI, 1985.

15. V. W. Guillemin and S. Sternberg, An algebraic model of transitive differential geometry, Bull. Amer. Math. Soc. 70 (1964), 16-47.

16. S. Mac Lane, Homology, Springer-Verlag, Berlin and New York, 1963. 
17. H. Matsumura, Commutative ring theory, Cambridge Univ. Press, London and New York, 1986.

18. J. P. May, Matric Massey products, J. Algebra 12 (1969), 533-568.

19. McCleary, User's guide to spectral sequences, Publish or Perish, Houston, TX, 1985.

20. M. Nakaoka, Homology of the infinite symmetric group, Ann. of Math. (2) 73 (1961), 229257.

21. D. Quillen, The spectrum of an equivariant cohomology ring, I, II, Ann. of Math. (2) 94 (1971), 549-602.

22. Ann. 194 (1971), 197-212.

23. $\ldots$ On the cohomology and $K$-theory of the general linear groups over a finite field, Ann. of Math. (2) 96 (1972), 552-586.

24. D. Ravenel, Complex cobordism and stable homotopy groups of spheres, Academic Press, San Diego, 1986.

25. J.-P. Serre, Algèbre locale-multiplicités, Lecture Notes in Math., vol. 11, Springer-Verlag, Berlin and New York, 1965.

26. R. P. Stanley, Invariants of finite groups and their applications to combinatorics, Bull. Amer. Math. Soc. 1 (1979), 475-511.

27. _ _ Hilbert functions of graded algebras, Adv. Math. 28 (1978), 57-83.

Mathematical Institute, 24-29 St. Giles, Oxford OX1 3LB, Great Britain

Current address: Department of Mathematics, University of Georgia, Athens, Georgia 30602

E-mail address: djb@sloth.math.uga.edu

Department of Mathematics, University of Georgia, Athens, Georgia 30602

E-mail address: jf cesloth.math.uga.edu 